\title{
Developmental Phase Transitions in Spatial Organization of Spontaneous Activity in Postnatal Barrel Cortex Layer 4
}

\author{
${ }^{\circledR}$ Shingo Nakazawa, ${ }^{1}{ }^{\circledR}$ Yumiko Yoshimura, ${ }^{2,3}$ Masahiro Takagi, ${ }^{2}$ Hidenobu Mizuno, ${ }^{4}$ and ${ }^{\circledR}$ Takuji Iwasato ${ }^{1,5}$ \\ ${ }^{1}$ Laboratory of Mammalian Neural Circuits, National Institute of Genetics, Mishima, Shizuoka, Japan, ${ }^{2}$ Division of Visual Information Processing, \\ National Institute for Physiological Sciences, Okazaki, Aichi, Japan, ${ }^{3}$ Department of Physiological Sciences, SOKENDAI (The Graduate University \\ for Advanced Studies), Okazaki, Aichi, Japan, ${ }^{4}$ Laboratory of Multi-Dimensional Imaging, International Research Center for Medical Sciences, \\ Kumamoto University, Kumamoto, Japan, and ${ }^{5}$ Department of Genetics, SOKENDAI (The Graduate University for Advanced Studies), Mishima, \\ Shizuoka, Japan
}

Spatially-organized spontaneous activity is a characteristic feature of developing mammalian sensory systems. However, the transitions of spontaneous-activity spatial organization during development and related mechanisms remain largely unknown. We reported previously that layer 4 (L4) glutamatergic neurons in the mouse barrel cortex exhibit spontaneous activity with a patchwork-type pattern at postnatal day $(\mathrm{P}) 5$, which is during barrel formation. In the current work, we revealed that spontaneous activity in mouse barrel-cortex L4 glutamatergic neurons exhibits at least three phases during the first two weeks of postnatal development. Phase I activity has a patchwork-type pattern and is observed not only at P5, but also P1, before barrel formation. Phase II is found at P9, by which time barrel formation is completed, and exhibits broadly synchronized activity across barrel borders. Phase III emerges around P11 when L4-neuron activity is desynchronized. The Phase I activity, but not Phase II or III activity, is blocked by thalamic inhibition, demonstrating that the Phase I to II transition is associated with loss of thalamic dependency. Dominant-negative (DN)-Racl expression in L4 neurons hampers the Phase II to III transition. It also suppresses developmental increases in spine density and excitatory synapses of L4 neurons in the second postnatal week, suggesting that Racl-mediated synapse maturation could underlie the Phase II to III transition. Our findings revealed the presence of distinct mechanisms for Phase I to II and Phase II to III transition. They also highlighted the role of a small GTPase in the developmental desynchronization of cortical spontaneous activity.

Key words: barrel cortex; development; layer 4; mouse; Rac1; spontaneous activity

Significance Statement

Developing neocortex exhibits spatially-organized spontaneous activity, which plays a critical role in cortical circuit development. The features of spontaneous-activity spatial organization and the mechanisms underlying its changes during development remain largely unknown. In the present study, using two-photon in vivo imaging, we revealed three phases (Phases I, II, and III) of spontaneous activity in barrel-cortex layer 4 (L4) glutamatergic neurons during the first two postnatal weeks. We also demonstrated the presence of distinct mechanisms underlying phase transitions. Phase I to II shift arose from the switch in the L4-neuron driving source, and Phase II to III transition relied on L4-neuron Racl activity. These results provide new insights into the principles of developmental transitions of neocortical spontaneous-activity spatial patterns.

Received May 9, 2020; revised July 27, 2020; accepted Aug. 16, 2020.

Author contributions: S.N. and T.I. designed research; S.N., Y.Y., M.T., and H.M. performed research; S.N. and Y.Y. analyzed data; S.N. and T.I. wrote the paper.

The authors declare no competing financial interests.

Acknowledgements: We thank Pierre Chambon for the $\mathrm{pCre-ER^{T2 }}$ vector; Klaus Hahn for pCyPet-Rac1 (T17N); Ryohei Yasuda for pCAG-CyRFP1 vector; Takuya Sato, Minako Kanbayashi, and Satoko Kouyama for technical assistance; Naoki Nakagawa and Luwei Wang for helping plasmid construction; and Iwasato laboratory members for critical reading of the manuscript and stimulating discussions.

This work was supported by KAKENHI Grants $16 \mathrm{H06460}$ (to Y.Y.) and $16 \mathrm{H06459}$ and $20 \mathrm{H03346}$ (to T.I.).

S. Nakazawa's present address: Department of Basic Neurosciences, University of Geneva, Geneva, Switzerland, 1211.

Correspondence should be addressed to Shingo Nakazawa at shingo.nakazawa@etu.unige.ch or Takuji Iwasato at tiwasato@nig.ac.jp.

https://doi.org/10.1523/JNEUROSCI.1116-20.2020

Copyright $\odot 2020$ the authors

\section{Introduction}

Synchronized spontaneous activity is a hallmark of mammalian sensory systems during early postnatal stages. It may support Hebbian-type synaptic competition to instruct neuronal circuit self-organization (Katz and Shatz, 1996; Yamamoto and LópezBendito, 2012; Kirkby et al., 2013; Ackman and Crair, 2014; Leighton and Lohmann, 2016). The spontaneous activity of the developing brain is extensively studied in the mammalian visual system. Wave-type propagation of spontaneous activity is observed in cultured retinas prepared from animals before eye opening (Meister et al., 1991). The retinal wave is transmitted to the visual cortex through the thalamus as well as to the superior 
colliculus in vivo (Hanganu et al., 2006; Ackman et al., 2012; Siegel et al., 2012). Accumulating evidence suggests a critical role for this patterned activity in visual circuit refinement (Stellwagen and Shatz, 2002; McLaughlin et al., 2003; Burbridge et al., 2014). Spontaneous activity with unique spatial organization has also recently been identified and characterized in other sensory systems of neonatal rodents (Tritsch et al., 2007; Golshani et al., 2009; Yang et al., 2013; Babola et al., 2018; Luhmann and Khazipov, 2018; Mizuno et al., 2018). We previously found a patchwork-type pattern in spontaneous activity of the neonatal mouse barrel cortex (Mizuno et al., 2018). In that study, we generated thalamocortical axon (TCA)-RFP transgenic mice exhibiting labeling of the barrel map, to precisely identify the barrel-cortex L4 in vivo. L4 glutamatergic neurons were labeled in these mice with GCaMP6s via in utero electroporation (IUE). Taking advantage of these two methods, we were able to analyze the spatial pattern of L4-neuron spontaneous activity in relation to the barrel map. L4 neurons within the same barrel fire together in the absence of sensory input at postnatal day $(\mathrm{P}) 5$, generating a barrel-corresponding patchwork-type pattern (Mizuno et al., 2018). At P11-P13, L4 glutamatergic neurons showed sparse spontaneous firing with no persisting patchwork-type patterns (Mizuno et al., 2018).

The current study extended our previous work and characterized the nature of spontaneous activity in L4 glutamatergic neurons of the barrel cortex in detail. We first asked the following two questions. (1) How is spontaneous activity in barrel-cortex L4 spatially organized during the developmental stage before barrel map formation? If the patchwork-type pattern of activity is important for barrel map formation, the observation of similar activity is expected before map formation. And (2) how does spontaneous-activity transition from patchwork-type activity at P5 to sparse-type activity at P11-P13? To address these questions, we conducted in vivo two-photon calcium imaging of L4 glutamatergic neurons in the mouse barrel cortex at several time points during the first two weeks of postnatal development. We found that at P1, even before the initiation of barrel formation, L4-neuron spontaneous activity showed a patchwork-type pattern. The presence of a novel type of spontaneous L4-neuron activity with a spatial pattern distinct from the patchwork-type and sparse-type ones was observed at P9. These observations suggest that L4-neuron spontaneous activity exhibits at least three phases during the first two weeks of postnatal development. Phase I included P1-P5 and exhibited a patchwork-type pattern. Phase II was detected around P9 and showed wide-area synchronization. Phase III included P11-P13 and showed sparse firing. Second, we investigated the mechanism underlying the Phase I to II transition. We found that Phase I activity, but not that of Phase II or III, depended on thalamocortical input. Thus, the Phase I to II transition was characterized by a shift in the activity source. Finally, we investigated the mechanism underlying the Phase II to III transition. We revealed that this transition required L4-neuron Rac1 activity. We also found that Rac1 activity was involved in the developmental increase of excitatory synapses on L4 neurons, which may underlie the Phase II to III transition. Thus, the results of this study provide dynamic and mechanistic insights into the spatial organization of spontaneous network activity during postnatal neuronal circuit maturation.

\section{Materials and Methods}

\section{Animals}

All experiments were performed according to the guidelines for animal experimentation of the National Institute of Genetics and the National
Institute of Physiologic Sciences and were approved by their animal experimentation committees. The day at which the vaginal plug was detected was designated as embryonic day (E)0 and E19 was defined as P0. For electrophysiological analysis, timed-pregnant ICR mice were obtained from SLC Japan (for naive) or CLEA Japan [for Tam control and dominant-negative (DN)-Rac1]. Sex of newborn mice was not identified.

Used transgenic lines are as follows: TCA-RFP (Mizuno et al., 2018), TCA-GFP (Mizuno et al., 2014), serotonin transporter (5-HTT)-Cre Tg208 (Arakawa et al., 2014), and R26-LSL-hM4Di- designer receptors exclusively activated by designer drugs (DREADD; JAX stock \#026219; Zhu et al., 2016). TCA-RFP mice were backcrossed from B6C3F2 to ICR three to seven times. TCA-GFP mice were backcrossed from B6 to ICR 11-13 times; 5-HTT-Cre and R26-LSL-hM4Di-DREADD mice were backcrossed from B6 to ICR one or two times.

\section{IUE}

IUE was conducted at E13 night or E14 morning. Timed-pregnant mice were anesthetized with an intraperitoneal injection of a combination anesthetic (medetomidine $(0.3 \mathrm{mg} / \mathrm{kg}$. Zenoaq, Domitor), midazolam $(4 \mathrm{mg} / \mathrm{kg}$. Maruishi Pharmaceutical, Dormicum), and butorphanol (5 mg/kg; Meiji Seika Pharma, Vetorphale) in saline, or with an intraperitoneal injection of pentobarbital $(50 \mathrm{mg} / \mathrm{kg}$; Kyoritsu Seiyaku, Somnopentyl) in saline and isoflurane inhalation (1-3.5\%; DS Pharma Animal Health, Zoetis). DNA solution (diluted in Milli-Q water and $<5 \%$ trypan blue; Sigma T8154) was injected into lateral ventricles of embryos via a pulled glass capillary (Drummond, 2-000-050), and two to five times square electric pulses $(40 \mathrm{~V} ; 50 \mathrm{~ms})$ were delivered by tweezer-electrodes (NepaGene, CUY650P5) and an electroporator (NepaGene, CUY21SC). When a combination anesthetic was used, atipamezole $(0.3 \mathrm{mg} / \mathrm{kg}$. Zenoaq, Antisedan) in saline was administered as an intraperitoneal injection after IUE.

\section{Dense GCaMP labeling}

pK152 $(1000 \mathrm{ng} / \mu \mathrm{l})$ was used. To identify in vivo imaged area in sections, pK036 $(5-15 \mathrm{ng} / \mu \mathrm{l})+$ pK037 $(1000 \mathrm{ng} / \mu \mathrm{l})$, or pK036 $(5-15 \mathrm{ng} / \mu \mathrm{l})+$ pK281 $(1000 \mathrm{ng} / \mu \mathrm{l})$, was also transfected as a marker of IUE.

Sparse GCaMP labeling (Supernova GCaMP)

pK031 (5-15 ng/ $\mu \mathrm{l})$ and pK175 (1000 ng/ $\mu \mathrm{l})$ were used. pK098 (500 ng/ $\mu l)$ was also transfected as a marker.

\section{DN-Rac1 histology and electrophysiology}

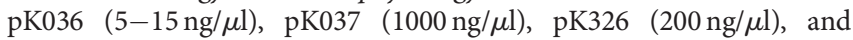
pK328 (1000 ng/ $\mu \mathrm{l})$ were used. For Tam control, pK036 (5-15 ng/ $\mu \mathrm{l})$ and pK037 (1000 ng/ $\mu \mathrm{l})$ were transfected.

\section{DN-Rac1 calcium imaging}

For DN-Rac1, Flpe-based Supernova-GCaMP6s [pK036 (5-15 ng/ $\mu \mathrm{l})$, pK313 (1000 ng/ $\mu \mathrm{l}), \mathrm{pK} 326(200 \mathrm{ng} / \mu \mathrm{l})$, and pK328 (1000 ng/ $\mu \mathrm{l})]$ were transfected. For Tam control, pK036 (5-15 ng/ $\mu \mathrm{l})$ and pK313 (1000 ng/ $\mu \mathrm{l})$ were transfected. As a marker of IUE, pK302 (200 ng/ $\mu \mathrm{l})$ or pK037 $(500 \mathrm{ng} / \mu \mathrm{l})$ was also transfected.

\section{Plasmids}

pK031: TRE-Cre (Mizuno et al., 2014);

pK036: TRE-Flpe-WPRE (Luo et al., 2016);

pK037: CAG-FRT-STOP-FRT-RFP-ires-tTA-WPRE (Luo et al., 2016);

pK098: CAG-loxP-STOP-loxP-nls-tagRFP-ires-tTA-WPRE (Luo et al., 2016);

pK152: CAG-GCaMP6s (Nakai et al., 2001; Chen et al., 2013; Mizuno et al., 2018);

pK175: CAG-loxP-STOP-loxP-GCaMP6s-ires-tTA-WPRE (Mizuno et al., 2018);

pK281: CAG-FRT-STOP-FRT-CyRFP-ires-tTA-WPRE;

pK302: CAG-tagBFP;

pK313: CAG-FRT-STOP-FRT-GCaMP6s-ires-tTA-WPRE;

pK326: CAG-ER ${ }^{\mathrm{T} 2} \mathrm{CreER}^{\mathrm{T} 2}-\mathrm{WPRE}$;

pK328: CAG-loxP-STOP-loxP-Rac1(T17N). 
pCAG-CyRFP was a gift from Ryohei Yasuda (Addgene plasmid \#84356; http://n2t.net/addgene:84356; RRID:Addgene_84356; Laviv et al., 2016). pTagBFP-actin vector was from Evrogen (\#FP174; Subach et al., 2008). pCre-ER ${ }^{\mathrm{T} 2}$ was a gift from Pierre Chambon (Feil et al., 1997; Indra et al., 1999; Li et al., 2000). pCyPet-Rac1(T17N) was a gift from Klaus Hahn (Addgene plasmid \#22784; http://n2t.net/addgene:22784; RRID:Addgene_22784; Machacek et al., 2009).

For construction of pK281, CyRFP excised with NheI and NotI from pCAG-CyRFP was blunted with SAP (Takara, 2660A) and Klenow (Takara, 2140A) and ligated into blunted SalI/EcoRV site of pK068 vectors (CAG-FRT-STOP-FRT-EGFP-ires-tTA-WPRE; Luo et al., 2016). For pK302, tagBFP excised with SalI/EcoRV from pK301 (CAG-loxPSTOP-loxP-tagBFP-ires-tTA-WPRE), for which tagBFP was cloned by PCR from pTagBFP-actin with primers KS130/KS131, was blunted with SAP and Klenow and ligated into blunted EcoRI site of pK038 vector (CAG-loxP-STOP-loxP-EGFP-ires-tTA-WPRE; Luo et al., 2016). For pK313, GCaMP6s was cloned by PCR from pK152 with primer pairs LW010/LW011 and was ligated into SalI/EcoRV site of pK068 vectors by using NEBuilder (New England BioLabs, E5520S). For pK326, we first constructed CAG-CreER ${ }^{\mathrm{T} 2}$. $\mathrm{CreER}^{\mathrm{T} 2}$ was cloned by PCR from pCreER $^{\text {T2 }}$ with primer pairs NN029/NN019 and ligated into SalI/NotI site of pK025 vector (CAG-turboRFP; Mizuno et al., 2010). Next, WPRE excised with NotI from pK068 was inserted into NotI site of CAG$\mathrm{CreER}^{\mathrm{T} 2}$. Finally, $\mathrm{ER}^{\mathrm{T} 2}$ fragment cloned by $\mathrm{PCR}$ with primer pairs NN030/NN031 was inserted into Sall site of CAG-CreER ${ }^{\mathrm{T} 2}$-WPRE. For pK328, Rac1(T17N) was cloned by PCR from pCyPet-Rac1(T17N) with primer pairs SN088/SN089 and was ligated into site of pK029 vectors (CAG-loxP-STOP-loxP-RFP-ires-tTA-WPRE; Mizuno et al., 2014) by using NEBuilder.

Primer sequences were as follows: KS130, CTGTCGACATGAGCG AGCTGATTAAGGAGA; KS131, AGATATCTTAATTAAGCTTGTG CCCCAGTTTG; LW010, GAATAGGAACTTCATGAGATCTCGCC ACCAT, LW011, TAACTCGATCTAGGATGCGGCCGCTCACTTC; NN019, CCCGCGGCCGCTCAAGCTGTGGCAGGGAAACCCT; NN029, GCTGTCGACAATTTACTGACCGTACACC; NN030, CCCGTCGA CGCCACCATGGCTGGAGACATGAGAGC; NN031, ATTGTCGAC AGCTGTGGCAGGGAAACCC; SN088, TATACGAAGTTATATGA TGCAGGCCATCAAGT; and SN089, ATCCTCGAGTCGCCGCTTA CAACAGCAGGCAT.

\section{Drug administration}

Clozapine-N-oxide (CNO; Sigma, C0832; Tocris, 4936) prepared in saline was intraperitoneally injected $(12 \mu \mathrm{g} / \mathrm{g}$ body weight; Hamm and Yuste, 2016). Lidocaine (AstraZeneca, Xylocaine) was subcutaneously injected (1\%, <10 $\mu$ l; Mizuno et al., 2018). Tamoxifen (Sigma, T5648) prepared in corn oil was intraperitoneally injected (50 $\mu \mathrm{g} / \mathrm{g}$; Guenthner et al., 2013).

\section{Craniotomy for in vivo imaging}

Cranial window for in vivo imaging was made as described (Nakazawa et al., 2018). Mice were anesthetized with isoflurane. The skin over the right hemisphere was removed using scissors to expose the skull, and Vetbond ( $3 \mathrm{M}, 1469)$ was applied to seal the incision. Whisker-related cortical area was detected with increases of GCaMP fluorescence induced by whisker stimulation. A small piece of bone covering labeled neurons was removed with a sterilized razor blade (Feather, FA-10) leaving the dura intact. Gelfoam (Pfizer) was used to stop bleeding as necessary. Cortex buffer (125 mm NaCl, $5 \mathrm{~mm} \mathrm{KCl,} 10 \mathrm{~mm}$ glucose, $10 \mathrm{~mm}$ HEPES, $2 \mathrm{~mm} \mathrm{CaCl}_{2}$, and $2 \mathrm{~mm} \mathrm{MgSO}_{4}$; pH 7.4; Holtmaat et al., 2009) was applied during opening the skull. The custom-made titanium bar ( $\mathrm{T}$ and I; Nakazawa et al., 2018) was glued to the skull near the window. A $2.5-\mathrm{mm}$ in diameter round cover glass (Matsunami, custom-made) was applied onto the exposed brain with $1 \%$ low melting point agarose (Sigma, A9793-100) in cortex buffer. The dental cement (GC Corporation, Unifast III) was applied to seal the cover glass and the titanium bar. For analgesic and anti-inflammation, carprofen $(5 \mathrm{mg} / \mathrm{kg}$, prepared in saline, Zoetis, Rimadyl) was subcutaneously injected.

\section{In vivo two-photon calcium imaging}

In vivo two-photon calcium imaging was performed under an unanesthetized condition. A heater was used to keep pups warm. Time-lapse images $(512 \times 512$ pixels, 12 bits $)$ were obtained at $1 \mathrm{~Hz}$ using a twophoton microscope (Zeiss, LSM 7MP) with W Plan-Apochromat $20 \times 1$ 1.0 DIC objective lens (Zeiss). Mai Tai eHP DeepSee titanium-sapphire laser (Spectra-Physics) running at 920 or $940 \mathrm{~nm}$ was used. Fluorescent proteins were simultaneously excited, and emitted fluorescence was filtered (500-550 nm for green and 575-620 nm for red) and detected with LSM BiG detectors (Zeiss). Higher signal-to-noise ratio images were obtained with 940 - or $960-\mathrm{nm}$ wavelength laser and a slower scan speed under isoflurane anesthesia to visualize the barrel position in TCA-RFP Tg mice. L4 was identified based on TCA-RFP signal and/or imaging depth. For $\mathrm{P} 4-\mathrm{P} 12$ mice, the presence of imaged neurons within L4 was confirmed by post hoc histologic analysis. For P1-P3 mice, because L4 is not clearly formed at these ages yet, imaging depths were determined based on dense GCaMP signals. Histologic analyses after the end of in vivo imaging also confirmed that imaged areas were located within the (prospective) large barrel field of the primary somatosensory cortex.

\section{Histology}

Mice were decapitated, and brains were fixed with $4 \%$ paraformaldehyde (PFA) in $0.1 \mathrm{M} \mathrm{PB}$ at $4^{\circ} \mathrm{C}$ for $1-2 \mathrm{~d}$. Right hemispheres were flattened and transferred to $2 \% \mathrm{PFA} / 30 \%$ sucrose in $0.1 \mathrm{M} \mathrm{PB}$ and kept at $4{ }^{\circ} \mathrm{C}$ for $1-2 \mathrm{~d}$. Tangential slices (100 $\mu$ m thick) were obtained with a ROM-380 freezing microtome (YAMATO, REM-710) and mounted with Antifade Mounting Medium (Longin et al., 1993). Slices which were not derived from TCA-GFP or TCA-RFP Tg were stained with anti-VGluT2 antibody (SYSY, 135403, 1:1000) and Alexa Fluor 647 goat anti-rabbit IgG (Invitrogen, A21244, 1:1000) and/or DAPI (Roche, 10236276001, $2 \mu \mathrm{g} / \mathrm{ml}$ ) before mounting to visualize barrel map. Images were acquired by a confocal microscope (Leica, TCS SP5). For quantitative analyses of dendritic spines, spiny stellate neurons located at the edge of large barrels $(\alpha-\delta, A-E$ arc $1-4)$ were chosen and their basal dendritic segments located inside the barrel were used. Spiny stellate neurons and barrel edges were identified as previously described (Mizuno et al., 2014). Confocal images were taken with $40 \times / 0.85$ objective lens, $1.2-\mu \mathrm{m}$ optical sectioning for measuring dendritic length, and with $63 \times / 1.3$ objective lens, $2 \times$ zoom, $0.1-\mu \mathrm{m}$ optical sectioning for spine count. Dendrites were cut into segments by a series of concentric circles from 10 to $70 \mu \mathrm{m}$ in radius with a $10-\mu \mathrm{m}$ interval. Segments which contained dendritic tip and those which were highly overlapped with other dendrites were excluded from spine count analysis. Filaments were classified as follows. If length $>5 \mu \mathrm{m}$ : dendritic branch. Else if head width $>0.6 \mu \mathrm{m}$ : mushroom. Else if length $>2 \mu \mathrm{m}$ : filopodium. Else: thin (Yuste and Bonhoeffer, 2004; Risher et al., 2014). Dendritic lengths and spine counts were measured on $2 \mathrm{D}$ images with using $3 \mathrm{D}$ images as references.

\section{Slice electrophysiology}

Whole-cell recording

For naive P9 and P11, non-labeled mice were used. For DN-Rac1 and Tam control, L4 glutamatergic neurons were sparsely labeled by the IUE-based Supernova-RFP transfection. Oblique coronal slices of barrel cortex (300 $\mu \mathrm{m}$ thick) were prepared from P9 or P11 mice under deep anesthesia with isoflurane and kept in a normal artificial cerebrospinal fluid containing the following: $126 \mathrm{~mm} \mathrm{NaCl}, 3 \mathrm{~mm} \mathrm{KCl}, 1.3 \mathrm{~mm} \mathrm{MgSO}_{4}$, $2.4 \mathrm{~mm} \mathrm{CaCl}_{2}, 1.2 \mathrm{~mm} \mathrm{NaH}_{2} \mathrm{PO}_{4}, 26 \mathrm{~mm} \mathrm{NaHCO}_{3}$, and $10 \mathrm{~mm}$ glucose, saturated with $95 \% \mathrm{O}_{2}$ and $5 \% \mathrm{CO}_{2}$. Non-labeled neurons (for naive P9 and P11) or RFP-positive neurons (for DN-Racl and Tam control at P11) in the barrel-cortex L4 were targeted by patch pipettes for wholecell recordings under fluorescent and infrared differential interference contrast optics (Olympus, BX51). The patch pipettes were filled with an internal solution containing the following: $130 \mathrm{~mm}$ K-gluconate, $8 \mathrm{~mm}$ $\mathrm{KCl}, 1 \mathrm{~mm} \mathrm{MgCl}$, $0.6 \mathrm{~mm}$ EGTA, 10 mм HEPES, 3 mм MgATP, $0.5 \mathrm{~mm}$ $\mathrm{Na}_{2} \mathrm{GTP}, 10 \mathrm{~mm}$ Na-phosphocreatine, and $0.2 \%$ biocytin (pH 7.3 with $\mathrm{KOH})$. To record miniature EPSCs (mEPSCs), tetrodotoxin $(1 \mu \mathrm{M}$, Abcam) and SR95531 (100 $\mu \mathrm{M}$, Abcam) were added to block action potentials and $\mathrm{GABA}_{\mathrm{A}}$ receptors, respectively. The membrane potentials 


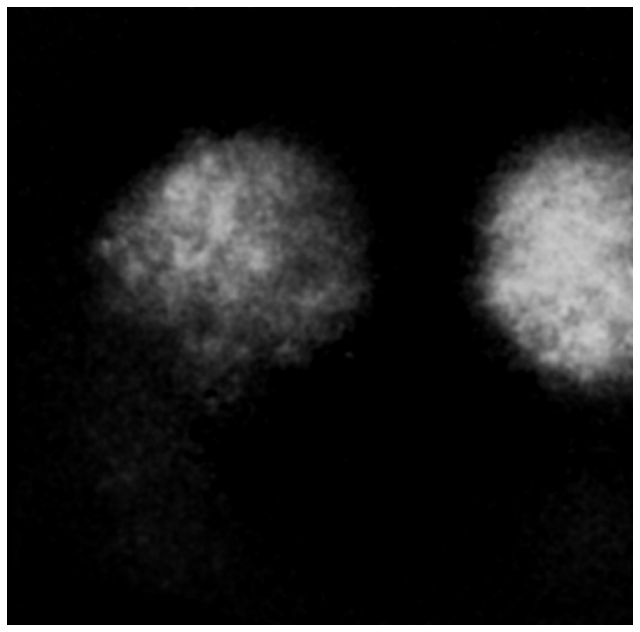

Movie 1. Spontaneous activity in the barrel cortex at P1. L4 neurons were densely labeled with CAG-GCaMP6s. [View online]

of recorded neurons were held at $-75 \mathrm{mV}$. Whole-cell recordings were performed and sampled using Axon Multiclamp 700B amplifier, Digidata 1440A, and pCLAMP10 software (Molecular Devices). Signals were sampled at $20 \mathrm{kHz}$. We selected cells with a high seal resistance $(>1 \mathrm{G} \Omega$ ) and a low series resistance $<30 \mathrm{M} \Omega$. mEPSCs were detected and analyzed using MiniAnalysis software (Synaptosoft).

Post hoc morphologic analysis

After electrophysiological recording, the slices were fixed with PFA in $0.1 \mathrm{M} \mathrm{PB}$ overnight at $4^{\circ} \mathrm{C}$ for biocytin staining. To visualize recorded neurons, slices were incubated with streptavidin conjugated to Alexa Fluor 488 (1:1000; Life Technologies) in $25 \mathrm{~mm}$ PBS containing $0.1 \%$ Triton X-100 overnight at $4^{\circ} \mathrm{C}$. Spiny stellate neurons were distinguished from other neurons by the spherical soma and the absence of a prominent apical dendrite (Staiger et al., 2004).

\section{In vivo image processing and quantification}

Preprocessing

Green channel was extracted and Gaussian filter $(\sigma=10$ pixels for the active contour analysis, 2 pixels for the others) was applied on Fiji/ ImageJ version 1.52p (Schindelin et al., 2012).

\section{Region of interest (ROI) setting}

Approximately $15 \times 15 \mu \mathrm{m}$ rectangular areas were selected. In sparse labeling experiments, ROIs were basically put on cell bodies, but some ROIs were put nearby the center of the cell bodies to avoid signal saturation which can be the cause of failure of signal detection.

\section{Signal detection and processing}

Intensity of the ROI was the mean of the pixels in the ROI. Raw calcium signals for each ROI, $\mathrm{F}(\mathrm{t})$, were converted to represent changes from baseline level, $\Delta \mathrm{F} / \mathrm{F}(\mathrm{t})$ defined as $\left(\mathrm{F}(\mathrm{t})-\mathrm{F}_{0}\right) / \mathrm{F}_{0}$, where $\mathrm{F}_{0}$ was the timeaveraged intensity at each ROI. $\Delta \mathrm{F} / \mathrm{F}(\mathrm{t})>1$ was counted as neuronal firing. The time period from when $\Delta \mathrm{F} / \mathrm{F}(\mathrm{t})$ became over the threshold to when $\Delta \mathrm{F} / \mathrm{F}(\mathrm{t})$ became lower than the threshold was defined as one firing events.

\section{Movie}

For Movies 1, 2, 3, $\mathrm{F}_{0}$ was subtracted from Gaussian filtered images, and brightness was adjusted for visualization. Movie is 10 times faster than the real time.

\section{Active contour drawing}

Images were binarized based on $\Delta \mathrm{F} / \mathrm{F}(\mathrm{t})$ threshold, and the area of the active contour was measured using Fiji/ImageJ. If a contour protruded from the view field, it was excluded from the analysis. When a contour

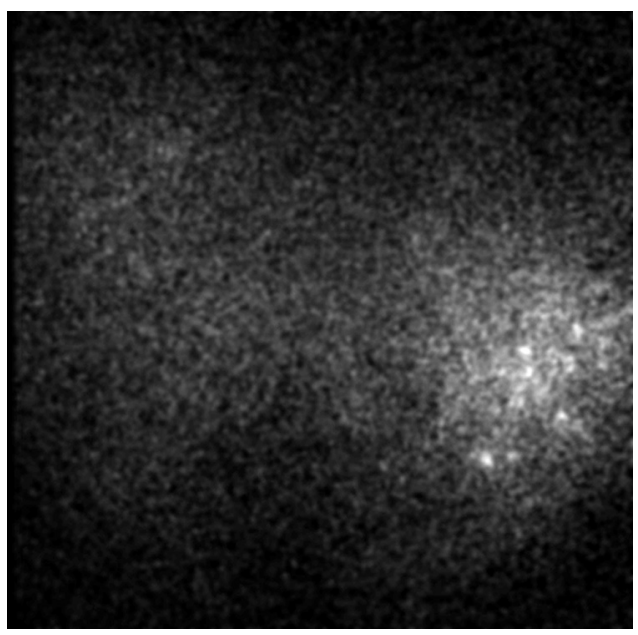

Movie 2. Spontaneous activity in the barrel cortex at P9. L4 neurons were densely labeled with CAG-GCaMP6s. [View online]

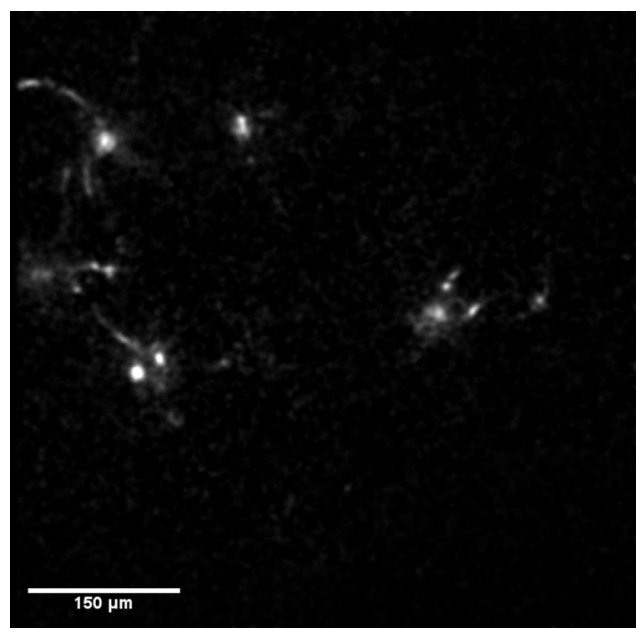

Movie 3. Spontaneous activity in the barrel cortex of Tam control and DN-Rac1 mice at P11. L4 neurons were sparsely labeled with Supernova-GCaMP6s. [View online]

was observed in the same position over multiple timeframes, only the contour on the initial timeframe was used for the analysis.

\section{Synchronized event detection}

Monte Carlo simulation was applied to infer the chance rate of ROI synchronization. For each ROI, firing periods and non-firing periods were calculated and they were randomly shuffled. After all ROIs underwent random shuffling process, the number of ROIs fired together was counted. This process was repeated 1000 times, and the distribution of active percentage in random firing was estimated as the proportion of the replications; 99th percentile of this distribution was used as the chance rate $(p<0.01)$. The time period in which the number of fired ROIs were over the chance rate was considered as a synchronized event.

\section{Statistics and computing}

Fiji/ImageJ ver. 1.52p (Schindelin et al., 2012) and custom-written scripts in Python 3.6.8 (Python Software Foundation) with its additional packages Numpy 1.16.4 (Oliphant, 2006; van der Walt et al., 2011), Scipy 1.2.1 (Virtanen et al., 2020), Matplotlib 3.1.0 (Hunter, 2007), Pandas 0.24.2 (McKinney, 2010), and OpenCV 3.3.1 (Bradski, 2000) were used to data analysis and visualization. Two-tailed Welch's $t$ test was used to test the differences among means unless otherwise noted. The asterisks and pound symbols in the figures indicate the following: ${ }^{\star} / \# p<0.05$,

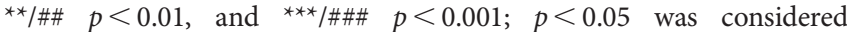


A

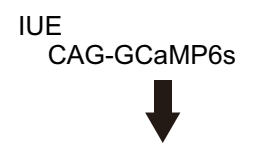

in vivo calcium imaging barrel cortex L4 P1, P3, or P5

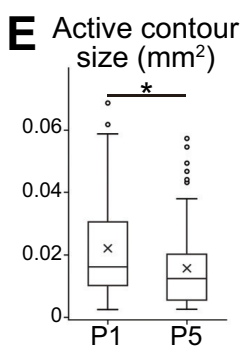

B
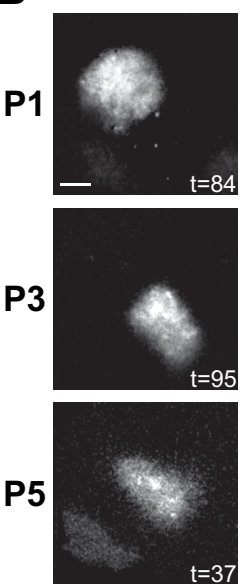

Representative time points
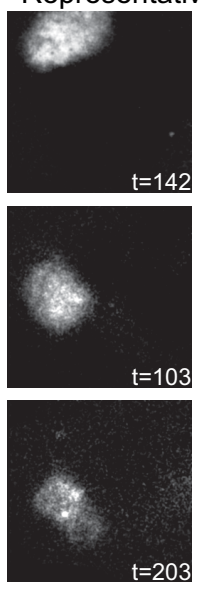
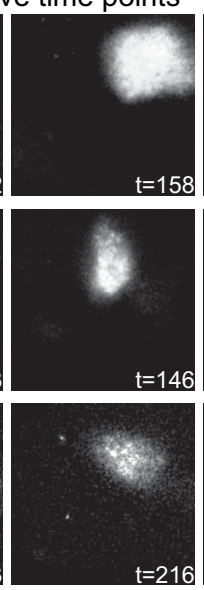
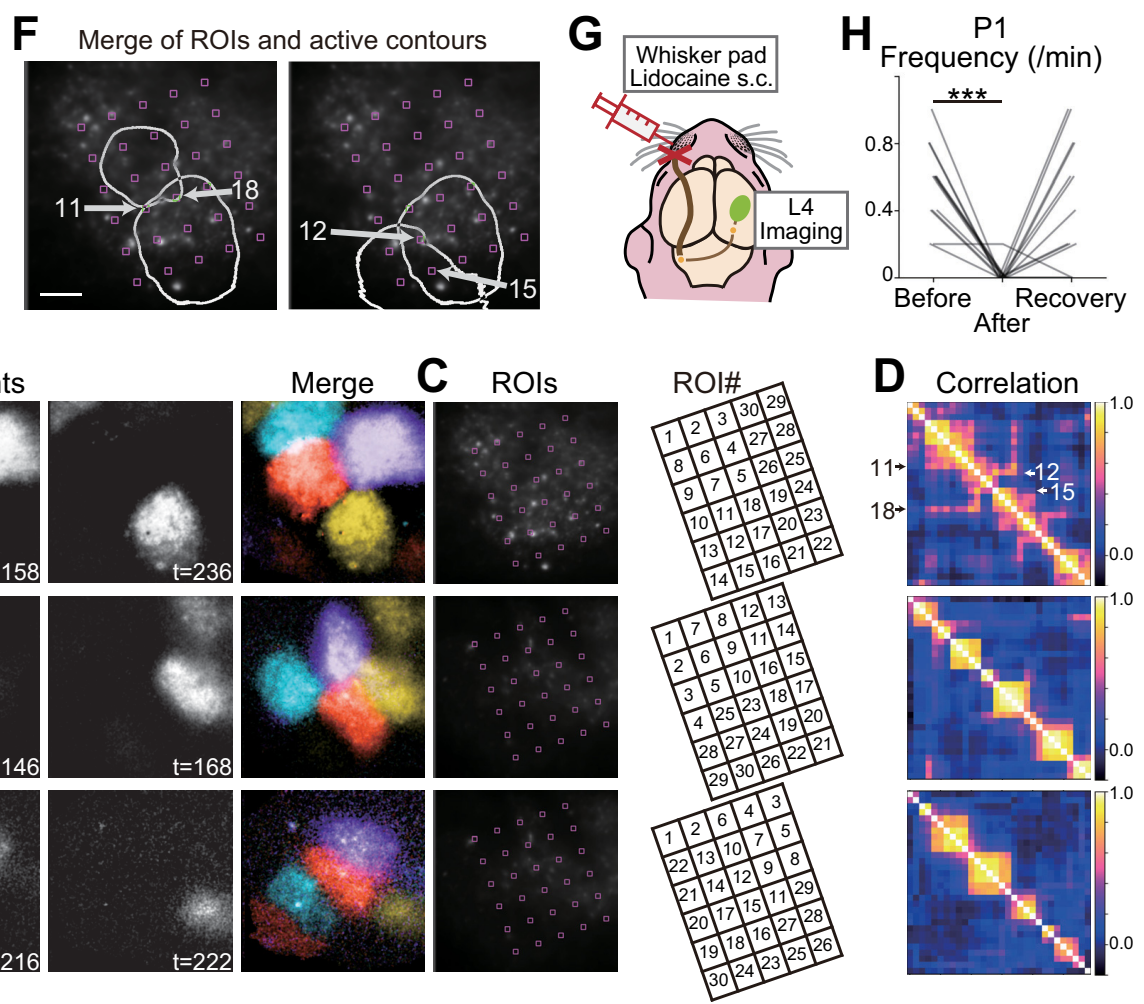

Figure 1. Patchwork-type spontaneous activity observed in L4 glutamatergic neurons of the barrel cortex in the first week of postnatal development. $\boldsymbol{A}$, Schematic of in vivo imaging with dense L4-neuron labeling. CAG-GCaMP6s plasmid vector was transfected by IUE. B, Representative examples of GCaMP6s signals at P1, P3, and P5. Brightness was adjusted for visualization. See also Movie 1. t, time (in seconds). C, left, ROls were placed in the barrel field uniformly, because barrels are not visible at P1 yet. Right, ROls were manually numbered to show the correlation clusters of Rols in $\boldsymbol{D}$ clearly. D, Correlation matrices of ROl pairs in $\boldsymbol{C}$. $\boldsymbol{E}$, Size of active contours at P1 was larger than that at P5. Box plot interpretation is described in Materials and Methods. $\boldsymbol{F}$, Two examples of overlapping active contours at P1. Arrows indicate ROls located on overlapping regions of neighboring contours. ROl numbers are the same as those shown in $\boldsymbol{C}$. $\boldsymbol{G}$, Schematic of the L4-neuron imaging experiment with peripheral silencing. $\boldsymbol{H}$, Local anesthetic lidocaine injection into the whisker pad significantly reduced the frequency of L4-neuron spontaneous activity in contralateral side of the barrel cortex at P1. Note that the activity was recovered in 15 min (recovery), suggesting that lidocaine injection did not damage the circuits. $\boldsymbol{B}, \boldsymbol{F}$, Scale bars, $100 \mu \mathrm{m} .{ }^{*} p<0.05,{ }^{* * *} p<0.001$.

statistically significant; $g$ indicates Hedges' $g$. In box plots, upper and lower limits of box represent 75 th and 25 th percentiles, crosses represent mean, horizontal lines represent median, upper and lower whiskers represent maximum and minimum within 1.5 interquartile range, and observations beyond the whisker range were marked with open circles as outliers. Sample size was described in Results. Representative examples of each figure were chosen from the following number of mice: Figure 1, five, three, and three mice at P1, P3, and P5, respectively; Figure 2, three and six mice at P5 and P9, respectively; Figure 3, three and four mice at P9 and P11-P12, respectively; Figure 6, four and five mice for Tam control and DN-Rac1, respectively. The following timeframes were used for each analysis: Figures $1 D, E, 2,3,240$ frames (for Fig. 3, P11, three mice: 180 frames) and Figures $1 H, 4,6,300$ frames.

Data and code availability

All data that support the conclusions and computational code used in the study will be available on manuscript publication.

\section{Results}

Patchwork-type pattern of L4-neuron spontaneous activity before barrel formation

We previously reported that spontaneous activity in barrel-cortex L4 shows barrel-corresponding patchwork pattern at P5 during the barrel formation period (Mizuno et al., 2018). If this pattern of spontaneous activity plays an important role in barrel circuit maturation, it is expected that similar activity would be observed before barrel formation. To examine this possibility, GCaMP6s was transfected into L4 glutamatergic neurons of the barrel cortex by IUE and calcium transients were acquired in vivo by two-photon microscopy at P1 and P3 (Fig. $1 A$ ). P5 mice were also analyzed as a known control. We found that, at P1 and P3, L4 neurons in the barrel cortex showed spontaneous activity with a similar spatial pattern to L4 neurons at P5 (Fig. 1B; Movie 1). When ROIs were uniformly placed in the barrel field, a group of ROIs located nearby showed high pairwise correlation with each other but low correlation with ROIs in different locations (Fig. 1C,D). Although the barrels are not visible at $\mathrm{P} 1$, it is likely that the spatial organization of the spontaneous activity found at P1 corresponds to the prospective barrel map. To characterize the spatial organization of L4-neuron spontaneous activity at P1 in more detail, we defined an active contour as the region where GCaMP fluorescence was over the threshold in a timeframe (for details, see Materials and Methods). An active contour at P5 largely corresponded to a barrel, although sometimes it corresponded to two or more barrels when neighboring barrels fired together. We found that the active contour size at P1 was slightly larger than that at P5 ( $p=0.011, t=2.564, g=0.418 ; n=76$ contours/ 3 mice at P1, 73 contours/3 mice at P5; Fig. $1 E$ ). Consistent with this result, at $\mathrm{P} 1$, neighboring contours showed some level of overlap (Fig. $1 F$ ). ROIs located on these overlapping regions showed high correlation coefficients with ROIs on each contour (Fig. 1D). These features of the activity pattern at P1 may reflect the fact that at this age TCA termini corresponding to different whiskers are not yet segregated (Erzurumlu and Gaspar, 2012; Mizuno et al., 2014). Electrophysiological studies also demonstrate that the functional whisker protomap at $\mathrm{P} 0-\mathrm{P} 1$ is not as 


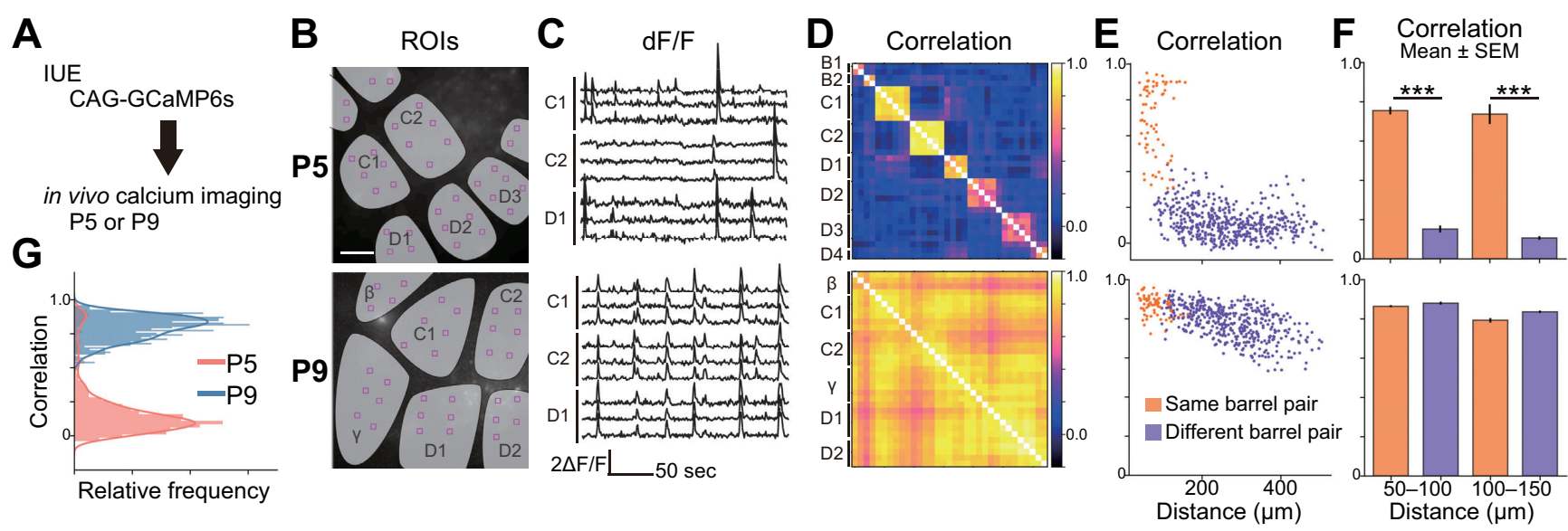

Figure 2. Spatial organization of L4-neuron spontaneous activity at $\mathrm{P} 9$ is different from that at P5. $\boldsymbol{A}$, Schematic of in vivo imaging with dense L4-neuron labeling. $\boldsymbol{B}$, ROls were placed on each barrel. Scale bar, $100 \mu \mathrm{m}$. C, Representative examples of in vivo calcium transients at P5 and P9. See also Movie 2. D, Pairwise ROl correlation matrices. $E$, Pairwise R0l correlation as a function of pair distance. $\boldsymbol{F}$, Comparison of correlation coefficients between ROI pairs from the same barrel (orange) and from different barrels (purple). Top, At P5, ROI pairs from the same barrels were highly correlated, and those located in different barrels showed low correlations even the distance between ROl pairs were similar. Bottom, At P9, ROI pairs were highly correlated regardless of whether they were in the same barrel or not. $\mathbf{G}$, Distribution of pairwise correlation coefficients at P5 and P9 shown in $\boldsymbol{D}$. P9 activity showed much higher synchrony than P5 activity. ${ }^{* * *} p<0.001$.

precise as later than P2-P3 (Mitrukhina et al., 2015), and oscillatory activity is altered between P0 and P7 (Yang et al., 2009), in the rat barrel cortex. The patchwork pattern could be refined at the same time as whisker-barrel circuit maturation during neonatal development. Taken together, these results suggest that L4-neuron spontaneous activity shows a patchwork-type pattern immediately after birth even before barrel formation, although the early patchwork pattern exhibits some distinct characteristics from the one at P5.

L4-neuron spontaneous activity at P5 is blocked by whiskerpad local anesthesia, suggesting that the activity originates from the periphery (Mizuno et al., 2018). To test whether P1 activity also originates from the periphery, we used the local anesthetic lidocaine in P1 mice (Fig. 1G). We found that lidocaine injection into whisker pad abolished L4-neuron activity in the contralateral barrel cortex at P1 $(p<0.001, t=8.101, g=3.177 ; n=13$ ROIs/2 mice; Fig. $1 H$ ). These results suggest that the periphery is the source of spontaneous activity in barrel-cortex L4 at P1, similar to $\mathrm{P} 5$.

\section{Wide-area synchronization of L4-neuron spontaneous activity at $\mathbf{P 9}$}

To investigate how spontaneous network activity transitions from patchwork-type at P1-P5 to sparse-type at P11-P13, we analyzed the spatial organization of L4-neuron spontaneous activity at P9. We first compared the activity pattern between P9 and P5 by transfecting GCaMP6s into a dense population of L4 glutamatergic neurons and performing calcium imaging in vivo (Fig. $2 A)$. ROIs were placed on barrels that were visualized using TCA-RFP Tg mice or post hoc staining with an anti-VGluT2 antibody and/or DAPI (Fig. 2B). At P5, L4 neurons showed spontaneous activity corresponding to the barrel map (Fig. 2C,D) as previously reported (Mizuno et al., 2018). ROIs put on the same barrels tended to fire together (Fig. 2C). Therefore, ROI pairs in the same barrels were highly correlated with each other, while ROIs located in different barrels tended to fire independently and showed low correlations (50-100 $\mu \mathrm{m}: p<0.001$, $t=23.024, g=3.798 ; n=84$ same barrel pairs and 39 different pairs; $100-150 \mu \mathrm{m}: p<0.001, t=12.369, g=4.842 ; n=21$ same and 128 different; 2 mice; Fig. 2D-F, top). While, at P9, L4- neuron spontaneous activity was widely synchronized in the barrel cortex across the barrel borders (Fig. 2C,D; Movie 2). They were still highly correlated even at large distance between ROI pairs (Fig. 2E) and regardless of whether ROI pairs were in the same barrel or not (50-100 $\mu \mathrm{m}: n=151$ same and 58 different pairs; $100-150 \mu \mathrm{m}$ : 50 same and 175 different pairs; three mice; Fig. 2F, bottom). Overall, L4 neurons at P9 showed much higher correlations than those at P5 (Fig. 2G). Thus, the spatial pattern of L4-neuron spontaneous activity at P9 was distinct from that at P5. Our analyses for P7 ( $n=3$ mice) identified both patchwork-type $(n=2$ mice) and widely synchronized-type ( $n=1$ mouse) activity, supporting that transition of two types of activity occurs between P5 and P9.

We next compared the P9 and P11-P12 activity patterns by using the Supernova method (Mizuno et al., 2014; Luo et al., 2016) to label a small population of L4 neurons with GCaMP6s and conducting in vivo calcium imaging in single-cell resolution (Fig. 3A). We previously reported that L4 neurons show asynchronous patterns of spontaneous activity at P11-P13 (Mizuno et al., 2018). Here, we confirmed that L4 neurons fire sparsely and show no clear spatial organization of spontaneous activity at $\mathrm{P} 11-\mathrm{P} 12$. At this age, pairwise correlation coefficients were low between ROIs placed on individual L4 neurons (Fig. 3B-D) and synchronized firing involving many neurons was not observed (Fig. 3E). In contrast, at P9, as observed in dense cell-labeling experiments (Fig. 2), L4 neurons showed high correlations even when they were in different barrels (Fig. $3 B-D$ ) and tended to fire synchronously (Fig. 3E). Overall, L4 neurons at P9 showed much higher correlations than those at P12 (Fig. 3F). Quantitative analyses revealed that the frequency of neuronal firing events was similar between P9 and P11-P12 [ $p=0.239, t=1.340$, $g=0.945 ; n=3$ (P9) and 4 (P11-P12) mice; Fig. 3G]. However, the ratio of synchronously firing events to total firing events was significantly higher at P9 than at P11-P12 $[p=0.009, t=4.170$, $g=3.012 ; n=3$ (P9) and 4 (P11-P12) mice; Fig. $3 H]$. Further, the peak height of synchronized events was significantly higher at P9 $(72 \pm 5 \%$, mean $\pm \mathrm{SD})$ than P11-P12 [26 $\pm 3 \% ; p=0.003$, $t=9.901, g=8.378 ; n=3$ (P9) and 4 (P11-P12) mice; Fig. $3 I]$. These results indicate that a large population of neurons distributed over multiple barrels fired together at P9. Thus, P9-type L4- 
A

IUE
Supernova GCaMP6s

in vivo calcium imaging $\mathrm{P} 9, \mathrm{P} 11$, or $\mathrm{P} 12$
J

\begin{tabular}{cccccc} 
P1 & P3 & P5 & P7 & P9 & P11 \\
\hline & Phase I & & Phase II & Phase III \\
\hline Patchwork & & & Synchronized & Sparse
\end{tabular}

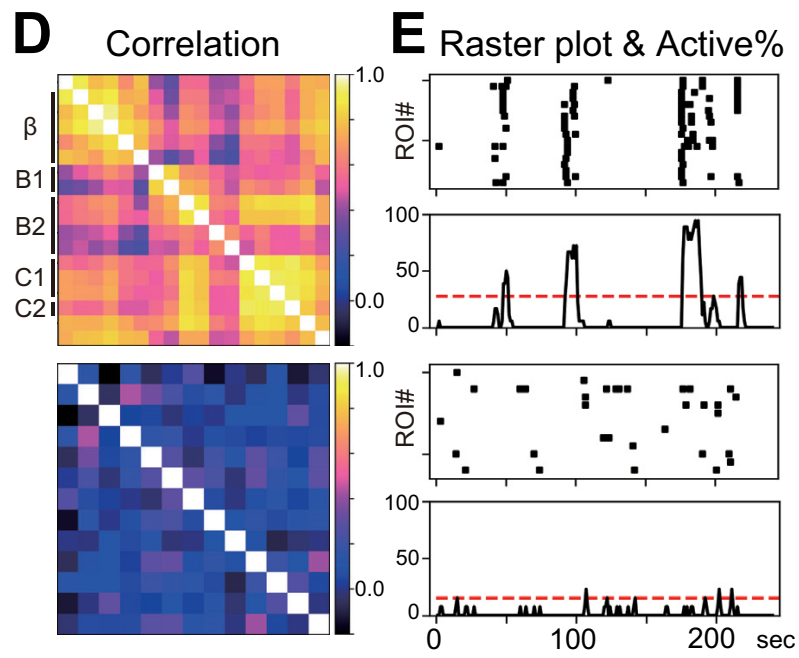

$\mathbf{H}_{\mathrm{s}}$

Ratio of synchronized events

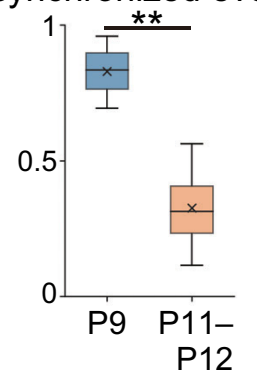

Peak height of synchronized events

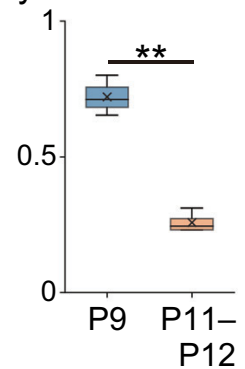

Figure 3. Spatial organizations of L4-neuron spontaneous activity in the barrel cortex at P9 and P11-P12. A, Schematic of in vivo calcium imaging with Supernova-mediated sparse L4-neuron GCaMPGs labeling. $\boldsymbol{B}$, ROls were placed on individual $L 4$ neurons. Scale bar, $100 \mu \mathrm{m}$. C, Representative examples of in vivo calcium transients. $\boldsymbol{D}$, Pairwise ROI correlation matrices. $\boldsymbol{E}$, Raster plots and active neuron ratio histograms. Dashed red lines indicate the chance rate $(p=0.01)$. $\boldsymbol{F}$, Distribution of pairwise correlation coefficients shown in $\boldsymbol{D}$. $\boldsymbol{G}$, Frequency of neuronal firing events. ROl averages of individual mice at P9 and P11-P12 were compared. $\boldsymbol{H}$, The peak height of synchronized events: the ratio of synchronized events to total firing events. R0I averages for individual mice were compared between P9 and P11-P12. I, The ratio of active ROls to total ROls in individual synchronized events. Maximum number of R0ls that fired together during individual synchronized events was divided by total ROI number, and averages for all synchronized events in individual mice were compared between P9 and P11-P12. J, Three phases of spontaneous network activity observed in barrel-cortex L4 neurons during the first two weeks of postnatal development. ${ }^{* *} p<0.01$.

neuron spontaneous activity is distinct from P11-P13-type activity.

These results suggest that there are at least three phases of spatial organization in L4-glutamatergic-neuron spontaneous activity during the first two weeks of postnatal development (Fig. 3J). Phase I is around P1 to P5 and shows a patchwork-type pattern. Phase II is around P9 and shows widely synchronized activity across the barrel borders. Phase III is around P11-P12 and shows sparse firing.

Ventrobasal (VB) thalamic inputs are dispensable for Phase II and Phase III activity

Phase I spontaneous activity is generated in the periphery and transmitted to cortical L4 along the ascending trigeminal pathway via the VB nucleus of the thalamus (Mizuno et al., 2018). Therefore, if VB neurons are silenced by inhibitory DREADD,
Phase I activity in cortical L4 is expected to be silenced. To test this possibility, we expressed hM4Di in the VB by crossing R26LSL-hM4Di-DREADD (Zhu et al., 2016) and 5-HTT-Cre Tg (Arakawa et al., 2014) mice. IUE was used to transfect L4 neurons in a dense population with GCaMP6s. We conducted in vivo two-photon calcium imaging of the barrel cortex of 5-HTTCre; R26-LSL-hM4Di-DREADD mice at P4 or P5 (Fig. 4A), and found that L4 spontaneous activity was silenced following CNO administration $(p<0.001, t=7.400, g=2.283 ; n=21$ ROIs $/ 3$ mice; Fig. $4 B$ ). It should be noted that, in 5-HTT-Cre Tg mice, Cre-mediated recombination is observed not only in the VB thalamus but also in a few other brain regions including the lateral geniculate nucleus (LGN) and medial geniculate nucleus (MGN) of the thalamus, raphe nuclei and deep layers of medial neocortex such as the cingulate (Arakawa et al., 2014). Although the neonatal barrel cortex is innervated by serotonergic axons 
originating from the raphe, these axons are sparse and not confined to L4 (Rebsam et al., 2002). Thus, it appears that silencing of Phase I L4 spontaneous activity in 5-HTT-Cre; R26-LSLhM4Di-DREADD mice is primarily attributed to silencing of VB inputs.

To examine whether Phase II and Phase III L4 spontaneous activity also depends on ascending trigeminal pathway via the $\mathrm{VB}$, we conduced calcium imaging of 5-HTT-Cre; R26-LSLhM4Di-DREADD mice at P9 (Phase II) and P11 (Phase III). L4-neuron spontaneous activity was not significantly changed between before and after $\mathrm{CNO}$ application $[p=0.928, t=0.091, g=$ $0.039 ; n=11$ ROIs $/ 2$ mice (Fig. $4 C$ ); $p=0.156, \quad t=1.471, \quad g=0.601 ; \quad n=12$ ROIs/2 mice (Fig. 4D)]. These results suggest that VB thalamic inputs have no or little contribution to Phase II and Phase III activity. Thus, the transition from Phase I to Phase II is associated with a switch in the source of spontaneous activity.

\section{DN-Racl suppresses increases in}

spine density during the second week of postnatal development

We next investigated the mechanism that controls the transition of Phase II to Phase III L4-glutamatergic-neuron spontaneous activity in the barrel cortex. Although sparse spontaneous activ-

ity is observed in various areas, layers and cell types of the mammalian cortex in the late developmental stage (Golshani et al., 2009; Rochefort et al., 2009; Colonnese et al., 2010; Luhmann and Khazipov, 2018; Modol et al., 2020), the underlying mechanisms of sparsification remain largely unresolved. Notably, little attention has been paid to the possible involvement of maturation of the excitatory system. Given that dendritic spine density dramatically increases in the second week of postnatal development (Blue and Parnavelas, 1983; Micheva and Beaulieu, 1996; De Felipe et al., 1997; Holtmaat and Svoboda, 2009), maturation of excitatory circuits may affect sparsification of L4-glutamatergic-neuron spontaneous activity. If this hypothesis is correct, blocking developmental spinogenesis could inhibit sparsification of L4-neuron spontaneous activity.

To test this hypothesis, we first examined whether spine density increased in barrel-cortex L4 glutamatergic neurons during the transition from Phase II to III. To reduce data dispersion, we focused our analyses on barrel-inner dendrites of barrel-edge spiny stellate neurons, which are the major type of L4 glutamatergic neurons in the barrel cortex (see Materials and Methods). We found that spine density more than doubled between P9 and P11, with increases in the mushroom and thin/stubby types [naive P9 vs P11; total: $p<0.001, t=11.770, g=1.533$; mushroom: $p<0.001, t=7.361, g=0.970$; thin/stubby: $p<0.001, t=10.488$, $g=1.362$. Welch's $t$ test with Holm correction; $n=102$ (P9) and 117 (P11) segments from 3 (P9) and 3 (P11) mice; Fig. 5A-C].

We used Racl (T17N), a DN-Rac1 that blocks spinogenesis in cultured hippocampal and cortical neurons (Penzes et al., 2003;
Hayashi-Takagi et al., 2010), to interfere with developmental spinogenesis. We used an inducible gene expression strategy to overexpress DN-Racl because constitutive DN-Racl expression interferes with radial migration of transfected cells (Kawauchi et al., 2003). IUE was used to transfect L4 glutamatergic neurons with tamoxifen-inducible Cre $\left(\mathrm{ER}^{\mathrm{T} 2} \mathrm{CreER}^{\mathrm{T} 2}\right.$; Feil et al., 1997; Casanova et al., 2002; Matsuda and Cepko, 2007) and Credependent DN-Rac1. Flpe-based Supernova RFP (Mizuno et al., 2014; Luo et al., 2016) was co-transfected for clear visualization of neuronal morphology. Then, tamoxifen was intraperitoneally injected at P7 and brain samples were collected at P11 (Fig. 5A). Comparing DN-Rac1 overexpression and tamoxifen control (Tam control) at P11 indicated that DN-Racl overexpression decreased spine densities (Tam control vs DN-Racl; total: $p<$ $0.001, t=4.308, g=0.564$; Fig. 5B,C). In DN-Racl-expressing neurons, mushroom-type spine density was also lower than in Tam control neurons [Tam control vs DN-Rac1; mushroom: $p<0.001, t=6.647, g=0.840$. Welch's $t$ test with Holm correction; $n=134$ (Tam) and 94 (DN) segments from 4 (Tam) and 3 (DN) mice; Fig. 5C]. This effect was more pronounced on the distal dendrites than in the proximal dendrites. On the proximal dendrites, mushroom-type spine density was decreased, but the total spine density was not changed in DN-Rac1-overexpressing neurons [mushroom: $p=0.004, t=3.068, g=0.911 ; n=23$ (Tam) and $20(\mathrm{DN})$ segments from 4 (Tam) and 3 (DN) mice; Fig. 5D]. On the distal dendrites, total spine and mushroom-type spine densities were decreased [total: $p=0.002, t=3.453, g=0.829$; Mushroom: $p=0.005, t=2.922, \mathrm{~g}=0.729$. Welch's $t$ test with Holm correction; $n=42$ (Tam) and $21(\mathrm{DN})$ segments from 4 
A

\begin{tabular}{lccc} 
& Naïve & Tam control & DN-Rac1 \\
\hline IUE & & & $\checkmark$ \\
Supernova RFP (Flpe-based) & $\checkmark$ & $\checkmark$ & $\checkmark$ \\
\hline CAG-ER ${ }^{\top 2}$ CreER ${ }^{\text {2 }}$ & & & $\checkmark$ \\
\hline CAG-LSL-DN-Rac1 & & & $\checkmark$ \\
\hline Tamoxifen i.p. at P7 & & $\checkmark$ & P11 \\
\hline Analysis & P9 or P11 & P11 & \\
\hline
\end{tabular}

B

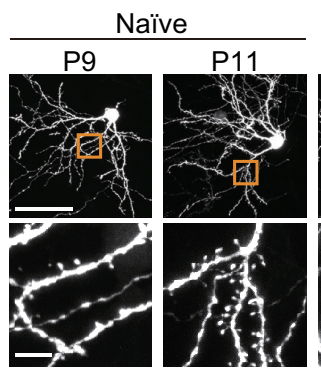

Tam cont. DN-Rac1

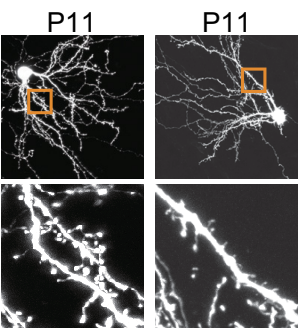

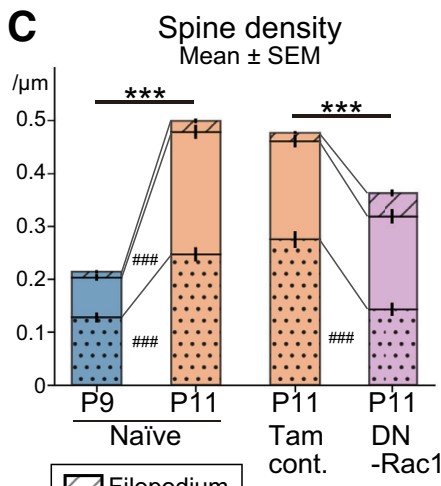
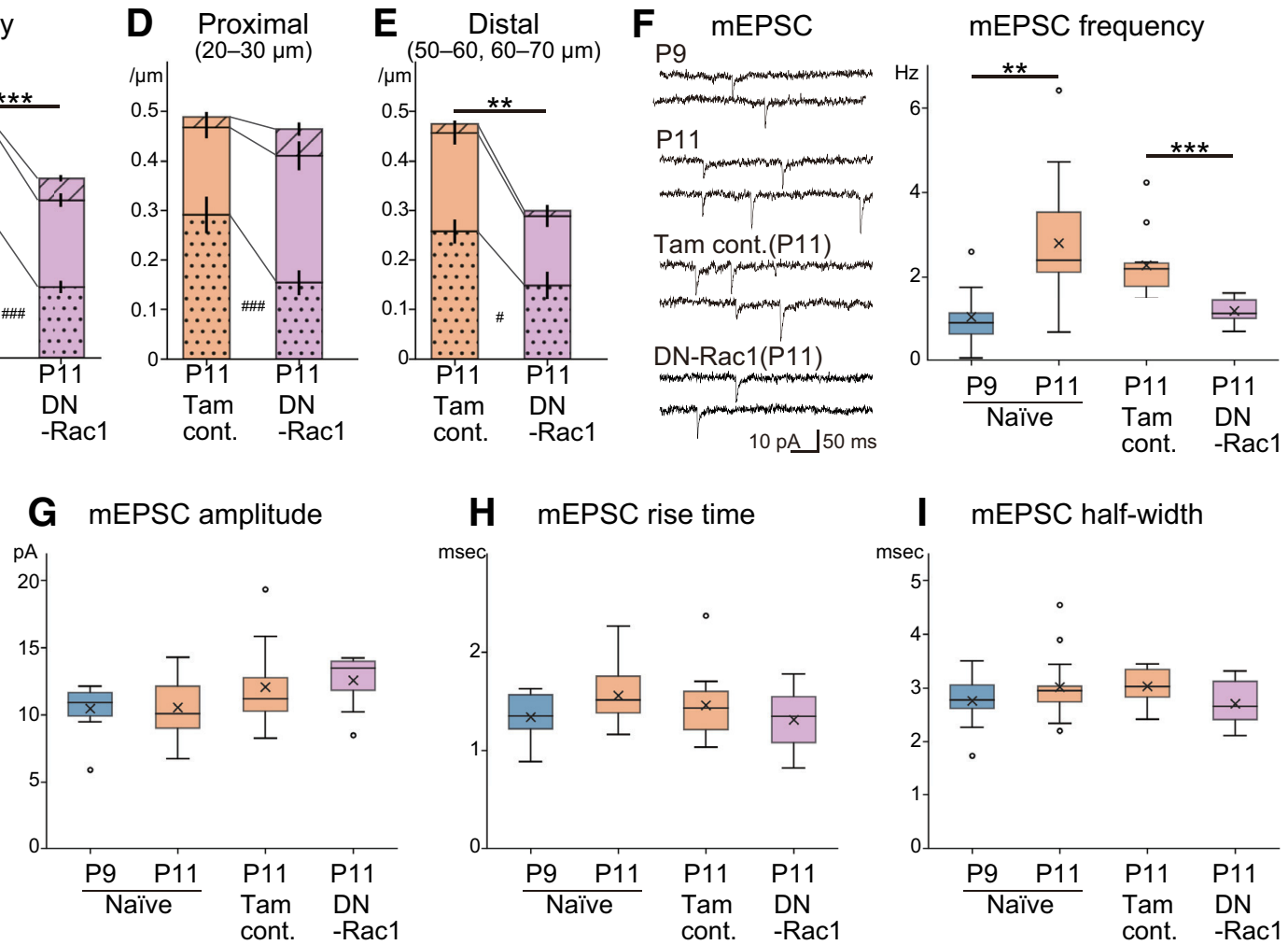

Figure 5. Dendritic spine maturation between Phase II and Phase III periods were interfered by DN-Rac1 expression in L4 neurons. A, Schematic of spine analysis experiments. L4 glutamatergic neurons were sparsely labeled with flpe-based Supernova RFP. For DN-Rac1, CAG-ER ${ }^{\mathrm{T} 2}$ CreER ${ }^{\mathrm{T2}}$ and CAG-LSL-DN-Rac1 plasmids were also transfected. For Tam control and DN-Rac1 mice, tamoxifen was administrated by intraperitoneal injection at P7. B, Representative examples of L4 spiny stellate neurons of naive P9, naive P11, Tam control P11, and DN-Rac1 P11. Scale bar, $50 \mu \mathrm{m}$ (top) and $5 \mu \mathrm{m}$ (bottom). C, Spine density of barrel-cortex L4 spiny stellate neurons of naive P11 was significantly higher than that of naive P9. Mushroom-type and thin/stubby-type spines increased from P9 to P11. DN-Rac1 neurons had significantly less spine density than Tam control neurons at P11. Mushroom-type spine density was also lower in DN than in Tam. D, On the proximal dendrites (20-30 $\mathrm{m}$ from soma center), total spine density of DN neurons was similar to that of Tam control at the same age (P11). However, mushroom-type spine density was lower in DN. $E$, On the distal dendrites $(50-60,60-70 \mu \mathrm{m})$, total spine density of DN was lower than that of Tam control, and mushroom-type spine density was also significantly lower in DN. $\boldsymbol{F}$, The average frequency of mEPSCS was significantly increased from P9 to P11 in naive animals. By overexpressing DN-Rac1, the average frequency of mEPSCs became lower than that of Tam control. The same experimental scheme shown in $\boldsymbol{A}$ was used for Tam control and DN-Rac1 of the physiological analysis. $\mathbf{G}-\mathbf{I}$, Additional properties of $\mathrm{mEPSCs}$. The amplitude (G), rise time $(\boldsymbol{H})$, and half-width $(\boldsymbol{I})$ of mEPSCs did not significantly change from P9 and P11 in naive mice, and also no significant differences between Tam control and DN-Rac1. \#p $<0.05$, ${ }^{* *} p<$ $0.01, * * * \# \# \# p<0.001$.

(Tam) and $3(\mathrm{DN})$ mice; Fig. 5E]. These results suggest that $\mathrm{DN}$ Racl overexpression inhibited increases in and maturation of dendritic spines between Phase II and Phase III.

We also assessed electrophysiological synaptic properties of DN-Rac1-overexpressing neurons. Like above, DN-Racl overexpression was induced by tamoxifen injection at P7, and $e x$ vivo whole-cell patch-clamp recordings were conducted at P11 (Fig. 5A). We analyzed mEPSCs recorded from L4 spiny stellate neurons identified by post hoc staining with biocytin. In naive animals, the frequency of mEPSCs increased from P9 to P11 (naive P9 vs P11: $p=0.002, t=3.633, g=1.396 ; n=10$ neurons/4 mice at P9, $n=13$ neurons/4 mice at P11; Fig. 5F). We found that the frequency was lower in DN-Racl-overexpressing cells compared with Tam controls at P11, which was consistent with our morphologic analysis of spine density (Tam control vs DN-Rac1: $p<0.001, t=4.522, g=1.809 ; n=$ 12 neurons $/ 4$ mice for Tam control, $n=10$ neurons $/ 5$ mice for DN-Rac1; Fig. 5F). There were no significant differences in the amplitude, rise time and half-width between them, suggesting that synaptic strength and kinetics did not change [naive P9 vs P11: $p=0.899, t=0.129, g=0.053$ (Fig. $5 G$ ); $p=0.065, t=1.951$, $g=0.802$ (Fig. $5 H$ ); $p=0.298, t=1.067, g=0.434$ (Fig. $5 I$ ); Tam control and DN-Racl: $p=0.654, t=0.455, g=0.188$ (Fig. $5 G$ ); $p=0.327, t=1.005, g=0.426$ (Fig. $5 H$ ); $p=0.075, t=1.900$, $g=0.835$ (Fig. 5I); $n=10$ neurons $/ 4$ mice at P9, $n=13$ neurons/4 mice at P11]. These results suggest that Racl inhibition reduces functional excitatory inputs to barrel-cortex L4 neurons. 
A

Tam control DN-Rac1

\begin{tabular}{lcc}
\hline IUE & & $\checkmark$ \\
Supernova GCaMP6s (Flpe-based) & $\checkmark$ & $\checkmark$ \\
\hline CAG-ER ${ }^{\text {22CreER }}$ T2 & & $\checkmark$ \\
\hline CAG-LSL-DN-Rac1 & $\checkmark$ & $\checkmark$ \\
\hline Tamoxifen i.p. at P7 & $\mathrm{P} 11$ & $\mathrm{P} 11$ \\
\hline in vivo calcium imaging & & \\
\hline
\end{tabular}

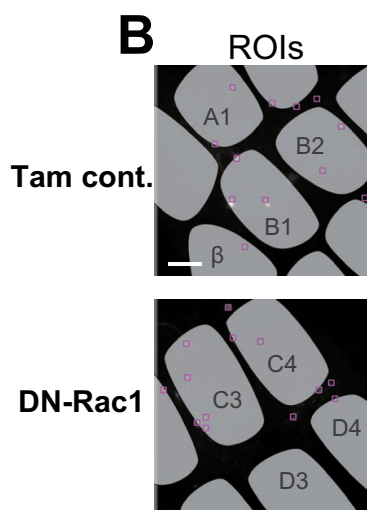

E Frequency of

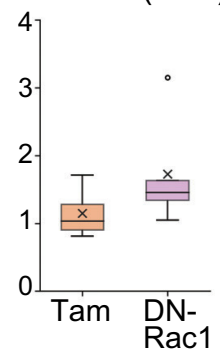

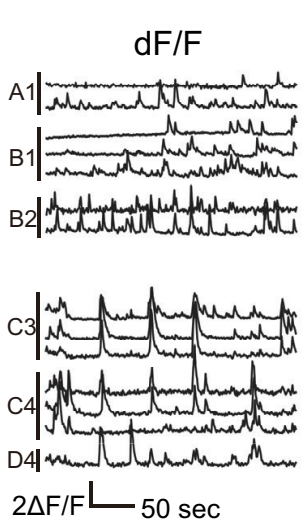

F Ratio of synchronized events

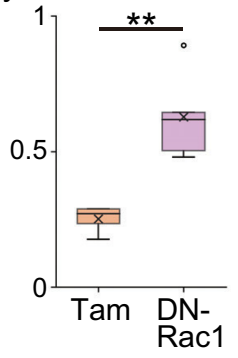

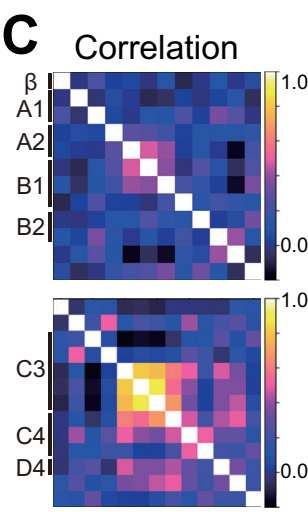

D Raster plot \& Active\%

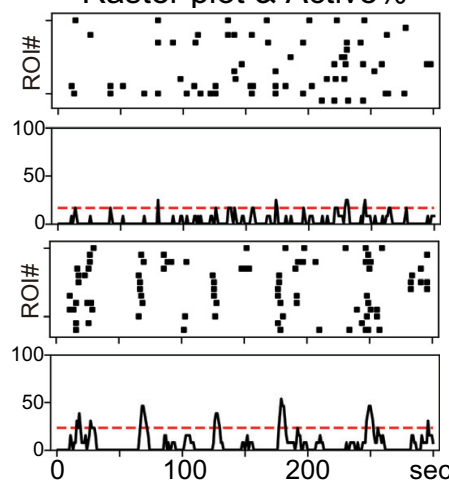

G Peak height of synchronized events

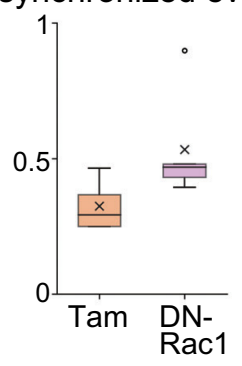

Figure 6. The Phase II to III transition was affected by DN-Rac1 overexpression in L4 glutamatergic neurons. A, Schematic of experiments. For DN-Rac1, Flpe-based Supernova-GCaMP6s, CAG-ER ${ }^{T 2}$ CreER ${ }^{T 2}$, and CAG-LSL-DN-Rac1 vectors were co-transfected into TCA-RFP mouse by IUE. $B$, Representative examples of in vivo calcium transients at P11. Scale bar, $100 \mu \mathrm{m}$. See also Movie 3. C, Pairwise ROl correlation matrices. $\boldsymbol{D}$, Raster plots and activity histograms. Dashed lines in activity histograms indicate chance rate $(p=0.01)$. $\boldsymbol{E}$, Frequency of neuronal firing events was similar between Tam control and DN-Rac1 mice. $\boldsymbol{F}$, The ratio of synchronized events ( $>$ chance rate) to total firing events was higher in DN-Rac1 than Tam control. $\mathbf{G}$, In average, $53 \pm 17 \%$ (mean \pm SD) of ROls were fired in individual synchronized events in DN-Rac1 mice, while, $32 \pm 8 \%$ in Tam control. ${ }^{* *} p<0.01$.

Taken together, these results suggest that Racl is important for increases in dendritic spine density and functional maturation of excitatory synapses at L4 glutamatergic neurons, which occur between the Phase II and Phase III periods.

\section{DN-Rac1 hampers the Phase II to III transition}

Finally, we conducted in vivo calcium imaging of DN-Racl-overexpressing L4 neurons. L4 neurons were co-transfected with $\mathrm{ER}^{\mathrm{T} 2} \mathrm{CreER}^{\mathrm{T} 2}$, Cre-dependent DN-Rac1, and SupernovaGCaMP6s. Tamoxifen was administrated at $\mathrm{P} 7$ and in vivo calcium imaging was conducted at P11 in single-cell resolution (Fig. 6A). As expected, Tam controls showed sparse firing (Fig. $6 B-D)$. Notably, we observed widely synchronized activity across the barrel borders in DN-Rac1 mice (Fig. 6B,C; Movie 3). Synchronized events were frequently observed in the activity histogram of DN-Rac1 mice (Fig. 6D). Quantitative analyses showed that the frequency of neuronal firing events was similar between Tam control and DN-Racl mice $[p=0.217, t=1.378$, $g=0.855 ; n=4$ (Tam control) and 5 (DN-Rac1) mice; Fig. 6E]. However, the ratio of synchronized events to total events was significantly higher in DN-Racl compared with Tam controls $[p=0.005, t=4.821, g=2.921 ; n=4$ (Tam control) and 5 (DNRac1) mice; Fig. $6 F$ ]. These results indicate that the synchronized events were prominent in DN-Racl cases. The ratio of active neurons to total neurons in individual synchronized events tended to be higher in DN-Racl than in Tam controls although it was not significantly different $[p=0.093, t=1.992, \mathrm{~g}=1.237$; $n=4$ (Tam control) and 5 (DN-Rac1) mice; Fig. 6G]. These features of L4-neuron spontaneous activity in DN-Racl mice at P11 were distinct from Phase III-type sparse firing in naive P11 mice and appeared similar to Phase II-type spontaneous activity (Fig. 3). However, it should be noted that DN-Racl activity at P11 was not identical to Phase II activity observed in naive P9 mice. For example, the ratio of synchronized events to total firing events was $83 \pm 9 \%$ (mean $\pm \mathrm{SD}$ ) for naive mice at P9 (Fig. $3 \mathrm{H}$ ), but $63 \pm 13 \%$ for DN-Rac1 mice at P11 (Fig. $6 F$ ). Also, the ratio of 
neurons involved in each synchronized event was $72 \pm 5 \%$ for naive mice at P9 (Fig. $3 I$ ), but $53 \pm 17 \%$ for DN-Racl mice at P11-P12 (Fig. 6G). IUE-mediated transfection, tamoxifeninduced Cre recombination, or DN-Racl effects may be insufficient to stop the phase transition completely. These results suggest that DN-Racl overexpression partially blocked the sparsification process that occurs during normal development between P9 and P11.

Taken together, these results reveal that Racl plays an important role in the Phase II to III transition of L4-glutamatergic-neuron spontaneous activity in the barrel cortex.

\section{Discussion}

Spatial organizations of spontaneous activity provide a template for activity-dependent development of mammalian neuronal circuits. We showed that there are at least three phases (I, II, and III) in spatial organization of barrel-cortex L4-glutamatergicneuron spontaneous activity during the first two postnatal weeks and further demonstrated mechanisms that are involved in Phase I to II and Phase II to III transitions.

\section{Phase I spontaneous activity of L4 glutamatergic neurons}

We previously reported that barrel-cortex L4 glutamatergic neurons exhibit patchwork-type spontaneous activity at P5 (Mizuno et al., 2018). Herein, we demonstrated that similar patchworktype spontaneous activity was present at P1 (Fig. 1), before barrel map formation. In the mouse barrel-cortex L4, thalamocortical connectivity is drastically reorganized to form the barrel map during the first postnatal week (Rice and Van der Loos, 1977; Erzurumlu and Jhaveri, 1990; Senft and Woolsey, 1991; Agmon et al., 1993; Rebsam et al., 2002; Lee et al., 2005; Mizuno et al., 2014; Nakazawa et al., 2018). The patchwork-type activity may play an important role in thalamocortical reorganization.

Thalamocortical slices prepared from mouse embryos exhibit wave-type spontaneous activity that propagates in the thalamus (Moreno-Juan et al., 2017; Antón-Bolaños et al., 2019). On the other hand, our analysis of cortical L4-neuron activity at P1 did not indicate the presence of wave-type activity. Similar to that observed at P5 (Mizuno et al., 2018), L4 activity at P1 was abolished by whisker-pad lidocaine administration (Fig. $1 G, H$ ), suggesting that Phase I activity arises from the periphery, rather than thalamus. The thalamic wave reported in the embryonic thalamus in vitro may not be present or sufficiently strong to induce L4-neuron calcium transients in the postnatal brain in vivo.

L2/3-neuron spontaneous activity was analyzed in the rat barrel cortex at P4-P7 (Golshani et al., 2009). These neurons exhibited Phase I-like activity, which was highly synchronous within local clusters. However, the spatial pattern of L2/3-neuron spontaneous activity does not track with specific barrel boundaries (Golshani et al., 2009). At this age, L4-neuron axons projecting to $\mathrm{L} 2 / 3$ are sparse and not confined within a barrel column (Bender et al., 2003). Such rudimentary axonal projections may explain the discrepancy between L4 and L2/3. It is unclear whether the patchwork pattern observed in L4 neurons at P1 corresponds to the barrel map because the barrel map does not exist at P1. A previous in vivo imaging study of the rat barrel cortex showed that the spontaneous firing clusters were similar to the cortical representations of whisker stimulation at P0-P1 (Yang et al., 2013). Technical improvement of the long-term in vivo imaging of the neonatal cortex (Nakazawa et al., 2018) would enable imaging starting at P1. Such studies will determine whether patchwork-type activity at P1 corresponds to the prospective barrel map. Spontaneous activity of GABAergic neurons in the neonatal mouse barrel cortex was recently reported (Modol et al., 2020). Further studies are required to fully understand the characteristics and interactions of Phase I-type locally clustered spontaneous activity among various cortical layers and cell-types in neonatal stages and their roles in somatosensory circuit maturation.

\section{Phase II spontaneous activity and Phase I to II transition}

During the Phase II periodaround P9, L4 glutamatergic neurons showed spontaneous activity that was widely synchronized across multiple barrels (Figs. 2, 3). The current study also found that the Phase I to II shift in L4-glutamatergic-neuron spontaneous activity arose from the switch in the L4-neuron driving source. Phase I L4-neuron activity was blocked by whisker-pad lidocaine administration and DREADD-induced thalamic inactivation (Figs. 1, 4), suggesting that this activity originates in the periphery and is relayed to L4 via TCAs. Conversely, Phase II and III activity failed to be blocked by DREADD-induced thalamic inactivation (Fig. 4), suggesting that these phases of L4 activity are delivered primarily from brain areas other than the VB thalamus. Similarly, a recent study reported that spontaneous activity in the mouse visual cortex loses dependence from the peripheral drive at the beginning of the second postnatal week (Gribizis et al., 2019).

The Phase I to II transition period is associated with thalamocortical connectivity maturation, e.g., the barrel map, representing whisker-related clusters of TCA termini, is established in the first postnatal week (Erzurumlu and Gaspar, 2012; Mizuno et al., 2014). Long-term potentiation is easily induced at thalamocortical synapses at P3-P7, but not P9-P11 (Lu et al., 2001). L4 neurons drastically reorganize their dendritic patterns postnatally, and by P9, they establish adult-type dendritic projections (Espinosa et al., 2009; Mizuno et al., 2014; Nakazawa et al., 2018). Subplate neuron neurites that initially accumulate in the barrel hollow gradually shift to the barrel septa between P6 and P10 (Piñon et al., 2009). The barrel net, which is the whiskerrelated axonal pattern of L2/3 neurons in the barrel-cortex L4, is not present at P6 but is formed by P10 (Sehara et al., 2010). Thus, thalamocortical and/or cortical maturation during these periods could be important for terminating the prevailing role of thalamocortical input and initiating the critical involvement of cortical or other non-thalamic input in driving L4-neuron spontaneous activity. Large neuronal ensembles of spontaneous activity that are similar in appearance to Phase II activity of L4 glutamatergic neurons were found in rat barrel-cortex L2/3 neurons between P8 and P11 (Golshani et al., 2009). In mouse, spontaneous-activity assemblies of GABAergic neurons become wider from P4-P6 to P7-P9 (Modol et al., 2020). However, it remains unknown whether the Phase II-like activity of L2/3 and GABAergic assemblies of the barrel cortex are independent of thalamic input.

\section{Phase II to III transition of L4-neuron spontaneous activity}

The adult neocortex exhibits spontaneous activity that is sparse and heterogeneously distributed in space and time across the neuronal population (Kerr et al., 2005; Wolfe et al., 2010). The sparseness of this neuronal activity allows precise information processing (Anderson et al., 2000; Chance et al., 2002; Wolfe et 
al., 2010). L4 glutamatergic neurons in the barrel cortex started to show sparse spontaneous activity by P11 (Fig. 3). Mice begin to show active whisking and explorative behavior around P14 (Arakawa and Erzurumlu, 2015; van der Bourg et al., 2017), which drastically increase the amount and complexity of information perceived and processed by L4 neurons. The developmental sparsification of L4 glutamatergic neurons may play a role in setting up the neural circuits that underlie adult barrelcortical function.

We provided evidence that the small GTPase Rac1, which is a key regulator of actin dynamics, plays an important role in the Phase II to III transition, a time point at which L4-neuron spontaneous activity undergoes sparsification. At P11, L4 glutamatergic neurons showed sparse-type (Phase III) spontaneous activity (Fig. 3). However, in P11 mice with L4 neurons that express DN-Rac1, L4 neurons exhibited spontaneous activity with a spatial pattern that was similar to the Phase II type (Fig. 6). Rac1 plays an important role in broad aspects of brain development, including cell migration, axon guidance and dendritic-spine formation, by regulating actin dynamics (Luo, 2000). During cortical development, the timing of spontaneous activity sparsification coincides with a drastic increase in dendriticspine density. We demonstrated that the spine densities of mouse barrel-cortex L4 glutamatergic neurons more than doubled between Phases II (P9) and III (P11; Fig. 5C). L4-neuron developmental spinogenesis was suppressed by DN-Rac1 expression (Fig. 5C). Furthermore, mEPSC frequency was lower in DN-Racl-expressing L4 neurons than in controls at P11 (Fig. 5F). These results suggest that Racl regulates the number of excitatory synapses on L4 glutamatergic neurons during the transition from Phase II to III. Thus, Racl may facilitate spontaneous-activity sparsification by promoting excitatory-network maturation in cortical L4.

Developmental sparsification of spontaneous activity is observed in the postnatal mammalian cortex across various areas, layers, cell types, and species including preterm human infants (Vanhatalo et al., 2005; Golshani et al., 2009; Rochefort et al., 2009; Colonnese et al., 2010; Iyer et al., 2015; Luhmann and Khazipov, 2018; Modol et al., 2020). However, the mechanisms underlying sparsification remain largely unknown. For example, the whisker plucking starting at P2 does not affect the L2/3-neuron spontaneous-activity sparsification in the rat barrel cortex, suggesting that sensory input is dispensable for this process (Golshani et al., 2009). To our knowledge, we provided the first evidence that the functional blockade of a cortical molecule affects the developmental sparsification of cortical spontaneous activity.

Our results do not exclude the possibility that inhibitory-circuit maturation plays a role in spontaneous-activity developmental sparsification. Symmetric synapses, which are putative inhibitory synapses, are increased (De Felipe et al., 1997), and intrinsic L2/3 excitability is decreased during the transition period (Golshani et al., 2009). Therefore, inhibitory-synapse maturation may also be involved in spontaneous-activity developmental sparsification. Previous experiments using dissociated neuronal cultures suggest that Racl affects $\mathrm{GABA}_{\mathrm{A}}$ receptor function by regulating its clustering and recycling (Meyer et al., 2000; Smith et al., 2014). Therefore, Rac1 may regulate L4-neuron spontaneous-activity sparsification by facilitating the maturation of both inhibitory and excitatory synapses.

Thus, these results suggest that Racl plays an important role in the Phase II to III transition of L4-glutamatergic-neuron spontaneous activity, possibly via the regulation of L4-neuron synaptic maturation.

\section{References}

Ackman JB, Crair MC (2014) Role of emergent neural activity in visual map development. Curr Opin Neurobiol 24:166-175.

Ackman JB, Burbridge TJ, Crair MC (2012) Retinal waves coordinate patterned activity throughout the developing visual system. Nature 490:219225.

Agmon A, Yang LT, O’Dowd DK, Jones EG (1993) Organized growth of thalamocortical axons from the deep tier of terminations into layer IV of developing mouse barrel cortex. J Neurosci 13:5365-5382.

Anderson JS, Lampl I, Gillespie DC, Ferster D (2000) The contribution of noise to contrast invariance of orientation tuning in cat visual cortex. Science 290:1968-1972.

Antón-Bolaños N, Sempere-Ferràndez A, Guillamón-Vivancos T, Martini FJ, Pérez-Saiz L, Gezelius H, Filipchuk A, Valdeolmillos M, López-Bendito G (2019) Prenatal activity from thalamic neurons governs the emergence of functional cortical maps in mice. Science 364:987-990.

Arakawa H, Erzurumlu RS (2015) Role of whiskers in sensorimotor development of C57BL/6 mice. Behav Brain Res 287:146-155.

Arakawa H, Suzuki A, Zhao S, Tsytsarev V, Lo FS, Hayashi Y, Itohara S, Iwasato T, Erzurumlu RS (2014) Thalamic NMDA receptor function is necessary for patterning of the thalamocortical somatosensory map and for sensorimotor behaviors. J Neurosci 34:12001-12014.

Babola TA, Li S, Gribizis A, Lee BJ, Issa JB, Wang HC, Crair MC, Bergles DE (2018) Homeostatic control of spontaneous activity in the developing auditory system. Neuron 99:511-524.e5.

Bender KJ, Rangel J, Feldman DE (2003) Development of columnar topography in the excitatory layer 4 to layer $2 / 3$ projection in rat barrel cortex. J Neurosci 23:8759-8770.

Blue ME, Parnavelas JG (1983) The formation and maturation of synapses in the visual cortex of the rat. II. Quantitative analysis. J Neurocytol 12:697712 .

Bradski G (2000) The OpenCV library. Dr Dobb's. J Software Tools 25:120125.

Burbridge TJ, Xu HP, Ackman JB, Ge X, Zhang Y, Ye MJ, Zhou ZJ, Xu J, Contractor A, Crair MC (2014) Visual circuit development requires patterned activity mediated by retinal acetylcholine receptors. Neuron 84:1049-1064.

Casanova E, Fehsenfeld S, Lemberger T, Shimshek DR, Sprengel R, Mantamadiotis T (2002) ER-based double iCre fusion protein allows partial recombination in forebrain. Genesis 34:208-214.

Chance FS, Abbott LF, Reyes AD (2002) Gain modulation from background synaptic input. Neuron 35:773-782.

Chen TW, Wardill TJ, Sun Y, Pulver SR, Renninger SL, Baohan A, Schreiter ER, Kerr RA, Orger MB, Jayaraman V, Looger LL, Svoboda K, Kim DS (2013) Ultrasensitive fluorescent proteins for imaging neuronal activity. Nature 499:295-300.

Colonnese MT, Kaminska A, Minlebaev M, Milh M, Bloem B, Lescure S, Moriette G, Chiron C, Ben-Ari Y, Khazipov R (2010) A conserved switch in sensory processing prepares developing neocortex for vision. Neuron 67:480-498.

De Felipe J, Marco P, Fairén A, Jones EG (1997) Inhibitory synaptogenesis in mouse somatosensory cortex. Cereb Cortex 7:619-634.

Erzurumlu RS, Jhaveri S (1990) Thalamic axons confer a blueprint of the sensory periphery onto the developing rat somatosensory cortex. Brain Res Dev Brain Res 56:229-234.

Erzurumlu RS, Gaspar P (2012) Development and critical period plasticity of the barrel cortex. Eur J Neurosci 35:1540-1553.

Espinosa JS, Wheeler DG, Tsien RW, Luo L (2009) Uncoupling dendrite growth and patterning: single-cell knockout analysis of NMDA receptor 2B. Neuron 62:205-217.

Feil R, Wagner J, Metzger D, Chambon P (1997) Regulation of Cre recombinase activity by mutated estrogen receptor ligand-binding domains. Biochem Biophys Res Commun 237:752-757.

Golshani P, Gonçalves JT, Khoshkhoo S, Mostany R, Smirnakis S, PorteraCailliau C (2009) Internally mediated developmental desynchronization of neocortical network activity. J Neurosci 29:10890-10899. 
Gribizis A, Ge X, Daigle TL, Ackman JB, Zeng H, Lee D, Crair MC (2019) Visual cortex gains independence from peripheral drive before eye opening. Neuron 104:711-723.e3.

Guenthner CJ, Miyamichi K, Yang HH, Heller HC, Luo L (2013) Permanent genetic access to transiently active neurons via TRAP: targeted recombination in active populations. Neuron 78:773-784.

Hamm JP, Yuste R (2016) Somatostatin interneurons control a key component of mismatch negativity in mouse visual cortex. Cell Rep 16:597-604.

Hanganu IL, Ben-Ari Y, Khazipov R (2006) Retinal waves trigger spindle bursts in the neonatal rat visual cortex. J Neurosci 26:6728-6736.

Hayashi-Takagi A, Takaki M, Graziane N, Seshadri S, Murdoch H, Dunlop AJ, Makino Y, Seshadri AJ, Ishizuka K, Srivastava DP, Xie Z, Baraban JM, Houslay MD, Tomoda T, Brandon NJ, Kamiya A, Yan Z, Penzes P, Sawa A (2010) Disrupted-in-Schizophrenia 1 (DISC1) regulates spines of the glutamate synapse via Rac1. Nat Neurosci 13:327-332.

Holtmaat A, Svoboda K (2009) Experience-dependent structural synaptic plasticity in the mammalian brain. Nat Rev Neurosci 10:647-658.

Holtmaat A, Bonhoeffer T, Chow DK, Chuckowree J, De Paola V, Hofer SB, Hübener M, Keck T, Knott G, Lee WC, Mostany R, Mrsic-Flogel TD, Nedivi E, Portera-Cailliau C, Svoboda K, Trachtenberg JT, Wilbrecht L (2009) Long-term, high-resolution imaging in the mouse neocortex through a chronic cranial window. Nat Protoc 4:1128-1144.

Hunter JD (2007) Matplotlib: a 2D graphics environment. Comput Sci Eng 9:90-95.

Indra AK, Warot X, Brocard J, Bornert JM, Xiao JH, Chambon P, Metzger D (1999) Temporally-controlled site-specific mutagenesis in the basal layer of the epidermis: comparison of the recombinase activity of the tamoxifen-inducible Cre-ER(T) and Cre-ER(T2) recombinases. Nucleic Acids Res 27:4324-4327.

Iyer KK, Roberts JA, Hellström-Westas L, Wikström S, Hansen Pupp I, Ley D, Vanhatalo S, Breakspear M (2015) Cortical burst dynamics predict clinical outcome early in extremely preterm infants. Brain 138:22062218.

Katz LC, Shatz CJ (1996) Synaptic activity and the construction of cortical circuits. Science 274:1133-1138.

Kawauchi T, Chihama K, Nabeshima Y, Hoshino M (2003) The in vivo roles of STEF/Tiam1, Rac1 and JNK in cortical neuronal migration. EMBO J 22:4190-4201.

Kerr JN, Greenberg D, Helmchen F (2005) Imaging input and output of neocortical networks in vivo. Proc Natl Acad Sci USA 102:14063-14068.

Kirkby LA, Sack GS, Firl A, Feller MB (2013) A role for correlated spontaneous activity in the assembly of neural circuits. Neuron 80:1129-1144.

Laviv T, Kim BB, Chu J, Lam AJ, Lin MZ, Yasuda R (2016) Simultaneous dual-color fluorescence lifetime imaging with novel red-shifted fluorescent proteins. Nat Methods 13:989-992.

Lee LJ, Iwasato T, Itohara S, Erzurumlu RS (2005) Exuberant thalamocortical axon arborization in cortex-specific NMDAR1 knockout mice. J Comp Neurol 485:280-292.

Leighton AH, Lohmann C (2016) The wiring of developing sensory circuitsfrom patterned spontaneous activity to synaptic plasticity mechanisms. Front Neural Circuits 10:71.

Li M, Indra AK, Warot X, Brocard J, Messaddeq N, Kato S, Metzger D, Chambon P (2000) Skin abnormalities generated by temporally controlled RXRalpha mutations in mouse epidermis. Nature 407:633-636.

Longin A, Souchier C, Ffrench M, Bryon PA (1993) Comparison of anti-fading agents used in fluorescence microscopy: image analysis and laser confocal microscopy study. J Histochem Cytochem 41:1833-1840.

Lu HC, Gonzalez E, Crair MC (2001) Barrel cortex critical period plasticity is independent of changes in NMDA receptor subunit composition. Neuron 32:619-634.

Luhmann HJ, Khazipov R (2018) Neuronal activity patterns in the developing barrel cortex. Neuroscience 368:256-267.

Luo L (2000) Rho GTPases in neuronal morphogenesis. Nat Rev Neurosci 1:173-180.

Luo W, Mizuno H, Iwata R, Nakazawa S, Yasuda K, Itohara S, Iwasato T (2016) Supernova: a versatile vector system for single-cell labeling and gene function studies in vivo. Sci Rep 6:35747.

Machacek M, Hodgson L, Welch C, Elliott H, Pertz O, Nalbant P, Abell A, Johnson GL, Hahn KM, Danuser G (2009) Coordination of Rho GTPase activities during cell protrusion. Nature 461:99-103.

Matsuda T, Cepko CL (2007) Controlled expression of transgenes introduced by in vivo electroporation. Proc Natl Acad Sci USA 104:1027-1032.
McKinney W (2010) Data structures for statistical computing in Python. Proceedings of the 9th Python in Science Conference, pp 51-56. Austin, Texas.

McLaughlin T, Torborg CL, Feller MB, O'Leary DD (2003) Retinotopic map refinement requires spontaneous retinal waves during a brief critical period of development. Neuron 40:1147-1160.

Meister M, Wong RO, Baylor DA, Shatz CJ (1991) Synchronous bursts of action potentials in ganglion cells of the developing mammalian retina. Science 252:939-943.

Meyer DK, Olenik C, Hofmann F, Barth H, Leemhuis J, Brünig I, Aktories K, Nörenberg W (2000) Regulation of somatodendritic GABAA receptor channels in rat hippocampal neurons: evidence for a role of the small GTPase Rac1. J Neurosci 20:6743-6751.

Micheva KD, Beaulieu C (1996) Quantitative aspects of synaptogenesis in the rat barrel field cortex with special reference to GABA circuitry. J Comp Neurol 373:340-354.

Mitrukhina O, Suchkov D, Khazipov R, Minlebaev M (2015) Imprecise whisker map in the neonatal rat barrel cortex. Cereb Cortex 25:34583467.

Mizuno H, Hirano T, Tagawa Y (2010) Pre-synaptic and post-synaptic neuronal activity supports the axon development of callosal projection neurons during different post-natal periods in the mouse cerebral cortex. Eur J Neurosci 31:410-424.

Mizuno H, Luo W, Tarusawa E, Saito YM, Sato T, Yoshimura Y, Itohara S, Iwasato T (2014) NMDAR-regulated dynamics of layer 4 neuronal dendrites during thalamocortical reorganization in neonates. Neuron 82:365-379.

Mizuno H, Ikezoe K, Nakazawa S, Sato T, Kitamura K, Iwasato T (2018) Patchwork-type spontaneous activity in neonatal barrel cortex layer 4 transmitted via thalamocortical projections. Cell Rep 22:123-135.

Modol L, Bollmann Y, Tressard T, Baude A, Che A, Duan ZRS, Babij R, De Marco García NV, Cossart R (2020) Assemblies of perisomatic GABAergic neurons in the developing barrel cortex. Neuron 105:93-105. e4.

Moreno-Juan V, Filipchuk A, Antón-Bolaños N, Mezzera C, Gezelius H, Andrés B, Rodríguez-Malmierca L, Susín R, Schaad O, Iwasato T, Schüle R, Rutlin M, Nelson S, Ducret S, Valdeolmillos M, Rijli FM, LópezBendito G (2017) Prenatal thalamic waves regulate cortical area size prior to sensory processing. Nat Commun 8:14172.

Nakai J, Ohkura M, Imoto K (2001) A high signal-to-noise $\mathrm{Ca}(2+)$ probe composed of a single green fluorescent protein. Nat Biotechnol 19:137141.

Nakazawa S, Mizuno H, Iwasato T (2018) Differential dynamics of cortical neuron dendritic trees revealed by long-term in vivo imaging in neonates. Nat Commun 9:3106.

Oliphant TE (2006) Guide to NumPy. Austin: Trelgol Publishing.

Penzes P, Beeser A, Chernoff J, Schiller MR, Eipper BA, Mains RE, Huganir RL (2003) Rapid induction of dendritic spine morphogenesis by transsynaptic ephrinB-EphB receptor activation of the Rho-GEF kalirin. Neuron 37:263-274.

Piñon MC, Jethwa A, Jacobs E, Campagnoni A, Molnár Z (2009) Dynamic integration of subplate neurons into the cortical barrel field circuitry during postnatal development in the Golli-tau-eGFP (GTE) mouse. J Physiol 587:1903-1915.

Rebsam A, Seif I, Gaspar P (2002) Refinement of thalamocortical arbors and emergence of barrel domains in the primary somatosensory cortex: a study of normal and monoamine oxidase a knock-out mice. J Neurosci 22:8541-8552.

Rice FL, Van der Loos H (1977) Development of the barrels and barrel field in the somatosensory cortex of the mouse. J Comp Neurol 171:545-560.

Risher WC, Ustunkaya T, Singh Alvarado J, Eroglu C (2014) Rapid Golgi analysis method for efficient and unbiased classification of dendritic spines. PLoS One 9:e107591.

Rochefort NL, Garaschuk O, Milos RI, Narushima M, Marandi N, Pichler B, Kovalchuk Y, Konnerth A (2009) Sparsification of neuronal activity in the visual cortex at eye-opening. Proc Natl Acad Sci USA 106:1504915054 .

Schindelin J, Arganda-Carreras I, Frise E, Kaynig V, Longair M, Pietzsch T, Preibisch S, Rueden C, Saalfeld S, Schmid B, Tinevez JY, White DJ, Hartenstein V, Eliceiri K, Tomancak P, Cardona A (2012) Fiji: an opensource platform for biological-image analysis. Nat Methods 9:676-682. 
Sehara K, Toda T, Iwai L, Wakimoto M, Tanno K, Matsubayashi Y, Kawasaki H (2010) Whisker-related axonal patterns and plasticity of layer 2/3 neurons in the mouse barrel cortex. J Neurosci 30:3082-3092.

Senft SL, Woolsey TA (1991) Growth of thalamic afferents into mouse barrel cortex. Cereb Cortex 1:308-335

Siegel F, Heimel JA, Peters J, Lohmann C (2012) Peripheral and central inputs shape network dynamics in the developing visual cortex in vivo. Curr Biol 22:253-258.

Smith KR, Davenport EC, Wei J, Li X, Pathania M, Vaccaro V, Yan Z, Kittler JT (2014) GIT1 and $\beta$ PIX are essential for GABA(A) receptor synaptic stability and inhibitory neurotransmission. Cell Rep 9:298-310.

Staiger JF, Flagmeyer I, Schubert D, Zilles K, Kötter R, Luhmann HJ (2004) Functional diversity of layer IV spiny neurons in rat somatosensory cortex: quantitative morphology of electrophysiologically characterized and biocytin labeled cells. Cereb Cortex 14:690-701.

Stellwagen D, Shatz CJ (2002) An instructive role for retinal waves in the development of retinogeniculate connectivity. Neuron 33:357-367.

Subach OM, Gundorov IS, Yoshimura M, Subach FV, Zhang J, Grüenwald D, Souslova EA, Chudakov DM, Verkhusha VV (2008) Conversion of red fluorescent protein into a bright blue probe. Chem Biol 15:11161124.

Tritsch NX, Yi E, Gale JE, Glowatzki E, Bergles DE (2007) The origin of spontaneous activity in the developing auditory system. Nature 450:5055.

van der Bourg A, Yang JW, Reyes-Puerta V, Laurenczy B, Wieckhorst M, Stüttgen MC, Luhmann HJ, Helmchen F (2017) Layer-specific refinement of sensory coding in developing mouse barrel cortex. Cereb Cortex 27:4835-4850. van der Walt S, Colbert SC, Varoquaux G (2011) The NumPy array: a structure for efficient numerical computation. Comput Sci Eng 13:22-30.

Vanhatalo S, Palva JM, Andersson S, Rivera C, Voipio J, Kaila K (2005) Slow endogenous activity transients and developmental expression of $\mathrm{K}+-\mathrm{Cl}$ cotransporter 2 in the immature human cortex. Eur J Neurosci 22:27992804.

Virtanen P, Gommers R, Oliphant TE, Haberland M, Reddy T, Cournapeau D, Burovski E, Peterson P, Weckesser W, Bright J, van der Walt SJ, Brett M, Wilson J, Millman KJ, Mayorov N, Nelson ARJ, Jones E, Kern R, Larson E, Carey CJ, et al. (2020) SciPy 1.0: fundamental algorithms for scientific computing in Python. Nat Methods 17:261-272.

Wolfe J, Houweling AR, Brecht M (2010) Sparse and powerful cortical spikes. Curr Opin Neurobiol 20:306-312.

Yamamoto N, López-Bendito G (2012) Shaping brain connections through spontaneous neural activity. Eur J Neurosci 35:1595-1604

Yang JW, Hanganu-Opatz IL, Sun JJ, Luhmann HJ (2009) Three patterns of oscillatory activity differentially synchronize developing neocortical networks in vivo. J Neurosci 29:9011-9025.

Yang JW, An S, Sun JJ, Reyes-Puerta V, Kindler J, Berger T, Kilb W, Luhmann HJ (2013) Thalamic network oscillations synchronize ontogenetic columns in the newborn rat barrel cortex. Cereb Cortex 23:12991316.

Yuste R, Bonhoeffer T (2004) Genesis of dendritic spines: insights from ultrastructural and imaging studies. Nat Rev Neurosci 5:24-34.

Zhu H, Aryal DK, Olsen RH, Urban DJ, Swearingen A, Forbes S, Roth BL, Hochgeschwender U (2016) Cre-dependent DREADD (designer receptors exclusively activated by designer drugs) mice. Genesis 54:439-446. 